\title{
A new insight into the consistency of the SPH interpolation formula
}

\author{
Leonardo Di G. Sigalottia,*, Otto Rendón ${ }^{\mathrm{b}, \mathrm{c}}$, Jaime Klapp ${ }^{\mathrm{d}}$, Carlos A. \\ Vargas $^{\mathrm{a}}$, Fidel Cruz ${ }^{\mathrm{a}}$ \\ ${ }^{a}$ Area of Physics of Irreversible Processes, Department of Basic Sciences, Autonomous \\ Metropolitan University - Azcapotzalco (UAM-A), Av. San Pablo 180, 02200 Mexico \\ City, Mexico \\ ${ }^{b}$ Center of Physics, Venezuelan Institute of Scientific Research (IVIC), Apartado Postal \\ 20632, Caracas 1020-A, Venezuela \\ ${ }^{c}$ Physics Department, Faculty of Science and Technology, University of Carabobo (UC), \\ Valencia, Carabobo State, Venezuela \\ ${ }^{d}$ Physics Department, National Institute of Nuclear Research (ININ), Carretera \\ Mexico-Toluca km. 36.5, La Marquesa, 52750 Ocoyoacac, State of Mexico, Mexico
}

\begin{abstract}
In this paper, the consistency of the smoothed particle hydrodynamics ( $\mathrm{SPH}$ ) interpolation formula is investigated by analytical means. A novel error analysis is developed in $n$-dimensional space using the Poisson summation formula, which enables the simultaneous treatment of both the kernel and particle approximation errors for arbitrary particle distributions. New consistency integral relations are derived for the particle approximation, which correspond to the cosine Fourier transform of the kernel consistency conditions. The functional dependence of the error bounds on the SPH interpolation parameters, namely the smoothing length, $h$, and the number of particles within the kernel support, $\mathcal{N}$, is demonstrated explicitly from which consistency conditions arise. As $\mathcal{N} \rightarrow \infty$, the particle approximation converges to the kernel approximation independently of $h$ provided that the particle mass scales with $h$ as $m \propto h^{\beta}$ with $\beta>n$, where $n$ is the spatial dimension. This implies that as $h \rightarrow 0$, the joint limit $m \rightarrow 0, \mathcal{N} \rightarrow \infty$, and $N \rightarrow \infty$
\end{abstract}

\footnotetext{
*Corresponding author

Email addresses: leonardo.sigalotti@gmail.com (Leonardo Di G. Sigalotti), ottorendon@gmail.com (Otto Rendón), jaime.klapp@inin.gob.mx (Jaime Klapp), carlovax@gmail.com (Carlos A. Vargas), fidelcru@gmail.com (Fidel Cruz)
} 
is necessary for complete convergence to the continuum, where $N$ is the total number of particles. The analysis also reveals a dominant error term of the form $(\ln \mathcal{N})^{n} / \mathcal{N}$ for finite $\mathcal{N}$, as it has long been conjectured based on the similarity between the SPH and the quasi-Monte Carlo estimates. When $\mathcal{N} \gg 1$, the error of the SPH interpolant decays as $\mathcal{N}^{-1}$ independently of the dimension. This ensures approximate partition of unity of the kernel volume.

Keywords: Particle methods; Numerical integration; Error analysis and interval analysis; Fourier transforms; Error bounds; Stability and convergence of numerical methods

\section{Introduction}

Smoothed particle hydrodynamics (SPH) is a Lagrangian particle method that was developed in the late 70 s for the simulation of astrophysical flows [1, 2]. However, it has emerged in recent years as a promising numerical technique for the simulation of complex fluid flows as well as for a large variety of problems in computational mechanics and related areas [3, 4, 5, 6]. Given the widespread use of SPH today, a complete understanding of the errors is mandatory to account for the lack of consistency of the standard SPH approximation. The mathematical concept of consistency is related to how closely the numerical discrete equations approximate the exact equations. In other words, consistency is a measure of the local truncation error. In $\mathrm{SPH}$ the discrete equations are assembled by replacing the field functions and gradients in the exact partial differential equations by their basic SPH interpolation formulae. Therefore, it is of interest to derive the functional dependence of their local truncation errors on the interpolation parameters, namely the smoothing length, $h$, and the number of neighbors within the kernel support, $\mathcal{N}$.

Although significant progress has been done over the years to restore SPH consistency (i.e., exact interpolation of low-order polynomials) [7, 8, 9, 10, 11, 12, 13] and investigate the truncation errors carried by the SPH summation interpolants [14, 15, 16, 17, 18, 19], their explicit functional dependence is not known and their actual nature is understood only in heuristic terms. For example, it is still unclear how the second-order accuracy noted by many authors for the continuous kernel approximation translates into the full discrete form, making a difficult task to provide simple general statements about the accuracy and convergence of the SPH interpolation. On the other hand, the 
convergence of SPH to the exact fluid-dynamics equations has been proved in Sobolev norms with respect to suitable regularizations of the pressure field [20] and with respect to the Wasserstein distance between measures as the number of particles tends to infinity [21, 22], while it has been proved numerically by using a modified transport-velocity formulation [23] and by relaxing the particle distributions toward satisfying partition of unity [12]. A consistent SPH approach for the simulation of interfacial multiphase flows has also recently appeared [24], which includes a surface tension formulation that reproduces the tangential properties of the tensor surface stress.

The development of the theory of meshless methods has been motivated by the fact that mesh-free and mesh-adaptive discretizations are often better suited to cope with geometric changes of the domain of interest, as for the case of free surfaces and large deformations, than the classical structured-mesh discretization techniques. Several mesh-free methods have been proposed since SPH was born. Among the most widely used in engineering applications, we find the element-free Galerkin method (EFG) [25], the reproducing kernel particle method (RKPM) [26], the moving least squares method (MLSM) 27], and their improved extensions GEFG, GRKPM [28, 29], and GMLSM [30], respectively. All these methods are based on a set of independent points (or particles) and use a shape (or kernel) function to approximate a continuous function and so they share similarities with the SPH method. The EFG method has been successfully used to solve a great number of problems in solid mechanics, with very few applications to fluid flow problems [31, 32]. In particular, the RKPM method has been used recently to explore the vibration performance of double-walled carbon nanotubes [33, 34, 35]. On the other hand, GRKPM simulations of concrete deterioration due to reinforcement corrosion has shown very good agreement with experimentally observed data [36]. Moreover, the dynamical response of multispan viscoelastic thin and deformable beams under the excitation of a moving mass has also been successfully studied using the GMLSM method [37, 38].

In this article we provide a new mathematical analysis to investigate the truncation errors carried by the SPH estimate of a function using the Poisson summation formula. The analysis can also be applied to evaluate the accuracy and convergence of other meshless methods as the ones mentioned above, including the quasi-Monte Carlo method. The Poisson formula was first used by Monaghan [3] in an attempt to estimate the errors in the SPH summation interpolant for a linear function in one dimension using equidistant particles and a Gaussian kernel. However, no conclusions were reached 
about the consistency of the method from his analysis. In contrast, here we provide expressions for the error bounds of the SPH estimate of an arbitrary function for non-uniformly distributed sets of particles. These expressions account for full consistency of the SPH summation interpolants and give the functional dependence of the error bounds on the SPH interpolation parameters. This article is organized as follows. Section 2 contains some preliminary definitions, while an overview of the SPH interpolation theory is given in Section 3, where key mathematical constructs are introduced for use in the error analysis. Section 4 deals with the error analysis of the SPH interpolation formula in one-space dimension $(n=1)$ and Section 5 extends the analysis to $n$-dimensions. Finally, a discussion of the results is given in Section 6 and the conclusions are summarized in Section 7.

\section{Preliminary definitions}

The Poisson summation formula is valid for all test functions $f(\mathbf{x}) \in$ $\mathcal{S}\left(\mathbb{R}^{n}\right)[39]$. Here $\mathbb{R}^{n}$ is the $n$-dimensional Euclidean space, where the length of vector $\mathbf{x}=\left(x_{1}, x_{2}, \ldots, x_{n}\right)$ is defined by the Euclidean norm $\|\mathbf{x}\|_{2}:=$

$\sqrt{x_{1}^{2}+x_{2}^{2}+\cdots+x_{n}^{2}}$. Let us also denote by $\mathbb{N}$ and $\mathbb{Z}$ the fields of all natural and integer numbers, respectively. The following definitions on the function spaces and compact support of a function are used.

Definition 1 (Continuous function spaces). $\mathcal{S}\left(\mathbb{R}^{n}\right)$ denotes the Schwartz space of all infinitely continuous functions on $\mathbb{R}^{n}$ with fast decay at infinity along with all derivatives.

Definition 2 (Dual function spaces). $\mathcal{S}^{\prime}\left(\mathbb{R}^{n}\right)$ is the dual space of $\mathcal{S}\left(\mathbb{R}^{n}\right)$, which is also a subspace of $\mathcal{D}^{\prime}\left(\mathbb{R}^{n}\right)$, i.e., the dual space of $\mathcal{D}\left(\mathbb{R}^{n}\right)$, which is the space of all smooth functions with compact support on $\mathbb{R}^{n}$. Every function of $\mathcal{D}$ belongs to $\mathcal{S}$.

Definition 3 (Compact support of a function). The support of a function $f(\mathbf{x}) \in \mathcal{D}\left(\mathbb{R}^{n}\right)$ that is locally integrable in $\mathbb{R}^{n}$ is the closure $\Gamma \in \operatorname{supp}(f)$ of the set of points $\mathbf{x}$ such that $f(\mathbf{x}) \neq 0$.

\section{SPH interpolation theory}

The SPH interpolation involves a two step procedure. The first is known as the kernel approximation and the second is known as the particle approx- 
imation [3].

\subsection{Kernel approximation}

Using ideas from distribution theory, the kernel approximation of a smooth function, $f(\mathbf{x}): \mathbb{R}^{n} \rightarrow \mathbb{R}$, is built up from the Dirac- $\delta$ sampling property by approximating the Dirac- $\delta$ distribution with a continuous kernel function $W$ [5] such that

$$
\langle f(\mathbf{x})\rangle=\int_{\Omega_{n}} f\left(\mathbf{x}^{\prime}\right) W\left(\left\|\mathbf{x}-\mathbf{x}^{\prime}\right\|, h\right) d^{n} \mathbf{x}^{\prime},
$$

where $\Omega_{n} \subset \mathbb{R}^{n}$ is the spatial domain and $h$ is the width of the kernel, most commonly known as the smoothing length. The notation $\langle f(\mathbf{x})\rangle$ is used to denote the kernel estimate of $f(\mathbf{x})$. The kernel function in relation (1) must fulfill the normalization condition

$$
M_{0}=\int_{\Omega_{n}} W\left(\left\|\mathbf{x}-\mathbf{x}^{\prime}\right\|, h\right) d^{n} \mathbf{x}^{\prime}=1
$$

and must be positive definite, symmetric, monotonically decreasing, and tend to $\delta\left(\mathbf{x}-\mathbf{x}^{\prime}\right)$ as $h \rightarrow 0$ so that $\langle f(\mathbf{x})\rangle \rightarrow f(\mathbf{x})$. Suitable kernels must also have a compact support so that $W=0$ if $\left\|\mathbf{x}-\mathbf{x}^{\prime}\right\|>k h$, where $k$ is some number that specifies the support of the kernel.

With the use of Taylor series expansions, many authors have noted that the kernel approximation (1) has a leading second-order error $O\left(h^{2}\right)$ when $h$ is not in the limit. If in the integral approximant (1) we expand $f\left(\mathbf{x}^{\prime}\right)$ in Taylor series about $\mathbf{x}$ and use relation (2), the kernel approximation becomes

$$
\langle f(\mathbf{x})\rangle=f(\mathbf{x})+\sum_{l=1}^{\infty} \frac{1}{l !} \nabla^{(l)} f(\mathbf{x}):: \cdots: \int_{\Omega_{n}}\left(\mathbf{x}^{\prime}-\mathbf{x}\right)^{l} W\left(\left\|\mathbf{x}-\mathbf{x}^{\prime}\right\|, h\right) d^{n} \mathbf{x}^{\prime}
$$

where $\nabla^{(l)}$ denotes the product of the $\nabla$ operator $l$ times with respect to coordinates $\mathbf{x}$, the symbol ":: ...:" denotes the $l$ th-order inner product, and $\left(\mathbf{x}^{\prime}-\mathbf{x}\right)^{l}$ is a tensor of rank $l$. From expansion (3) it follows that exact interpolation of a polynomial of order $m$ (i.e., consistency $C^{m}$ ) can be obtained if the family of consistency relations (or moments of the kernel) [11]

$$
\mathbf{M}_{l}=\int_{\Omega_{n}}\left(\mathbf{x}^{\prime}-\mathbf{x}\right)^{l} W\left(\left\|\mathbf{x}-\mathbf{x}^{\prime}\right\|, h\right) d^{n} \mathbf{x}^{\prime}=\mathbf{0}^{(l)},
$$

are exactly fulfilled for $l=1,2, \cdots, m$, where $\mathbf{0}^{(1)}=(0,0,0)$ is the null vector and $\mathbf{0}^{(l)}$ is the zero tensor of rank $l$. $C^{0}$ consistency of the kernel 
approximation is always guaranteed because of the normalization condition (2), while relations (4) are always satisfied for $l=1$ due to the symmetry of the kernel and therefore $C^{1}$ consistency is also automatically ensured. The same is true for all odd $l \geq 3$. Only for $l$ even the integrals (4) contribute with finite sources of error unless $W\left(\left\|\mathbf{x}-\mathbf{x}^{\prime}\right\|, h\right) \rightarrow \delta\left(\mathbf{x}-\mathbf{x}^{\prime}\right)$. The second-order error follows from the $l=2$ non-vanishing term in expansion (3). Using Eq. (1) it follows that [40]

$$
\mathbf{M}_{2}=\langle\mathbf{x x}\rangle-\langle\mathbf{x}\rangle\langle\mathbf{x}\rangle \neq \mathbf{0}^{(2)}
$$

provided that consistencies $C^{0}$ and $C^{1}$ are achieved. This term is just the variance of the position of the interpolation points (particles) and is a measure of the spread in position relative to the mean. Thus $C^{2}$ consistency is not achieved by the kernel approximation unless $W\left(\left\|\mathbf{x}-\mathbf{x}^{\prime}\right\|, h\right) \rightarrow \delta\left(\mathbf{x}-\mathbf{x}^{\prime}\right)$. The form of the second moment in (5) bears a tight resemblance to the expectation value derived by Di Lisio, Grenier, and Pulvirenti [21] for the SPH convergence of a sequence of empirical measures when $N \rightarrow \infty$. However, note that upon choosing a kernel function with vanishing $\mathbf{M}_{2}, C^{2}$ consistency can be achieved for the kernel approximation.

A similar analysis for the kernel estimate of the gradient, namely

$$
\langle\nabla f(\mathbf{x})\rangle=\int_{\Omega_{n}} f\left(\mathbf{x}^{\prime}\right) \nabla W\left(\left\|\mathbf{x}-\mathbf{x}^{\prime}\right\|, h\right) d^{n} \mathbf{x}^{\prime},
$$

leads to the Taylor series expansion

$$
\langle\nabla f(\mathbf{x})\rangle=\sum_{l=0}^{\infty} \frac{1}{l !} \nabla^{(l)} f(\mathbf{x}):: \cdots: \int_{\Omega_{n}}\left(\mathbf{x}^{\prime}-\mathbf{x}\right)^{l} \nabla W\left(\left\|\mathbf{x}-\mathbf{x}^{\prime}\right\|, h\right) d^{n} \mathbf{x}^{\prime},
$$

where the moments of the kernel gradient must satisfy the following conditions to achieve $C^{m}$ consistency

$$
\begin{aligned}
& \mathbf{M}_{0}^{\prime}=\int_{\Omega_{n}} \nabla W\left(\left\|\mathbf{x}-\mathbf{x}^{\prime}\right\|, h\right) d^{n} \mathbf{x}^{\prime}=\mathbf{0}^{(1)} \\
& \mathbf{M}_{1}^{\prime}=\int_{\Omega_{n}}\left(\mathbf{x}^{\prime}-\mathbf{x}\right) \nabla W\left(\left\|\mathbf{x}-\mathbf{x}^{\prime}\right\|, h\right) d^{n} \mathbf{x}^{\prime}=\mathbf{I} \\
& \mathbf{M}_{l}^{\prime}=\int_{\Omega_{n}}\left(\mathbf{x}^{\prime}-\mathbf{x}\right)^{l} \nabla W\left(\left\|\mathbf{x}-\mathbf{x}^{\prime}\right\|, h\right) d^{n} \mathbf{x}^{\prime}=\mathbf{0}^{(l+1)}
\end{aligned}
$$

for $l=2,3, \cdots, m$, where $\mathbf{I}$ is the unit tensor. 


\subsection{Particle approximation}

If the spatial domain $\Omega_{n}$ is divided into $N$ sub-domains, labeled $\Omega_{a}$, each of which encloses an interpolation point (or particle) $a$ at position $\mathbf{x}_{a} \in \Omega_{a}$, the discrete equivalent of Eq. (1) is defined by

$$
f_{a}=\sum_{b=1}^{\mathcal{N}} f_{b} W_{a b} \Delta V_{b},
$$

where $W_{a b}=W\left(\left\|\mathbf{x}_{a}-\mathbf{x}_{b}\right\|, h\right), \Delta V_{b}$ is the volume of sub-domain $\Omega_{b}$, and the summation is over $\mathcal{N}$ points within the support of the kernel of spherical volume $\mathcal{V}_{n}=B_{n}(k h)^{n} / n$, where $B_{n}=2 \pi^{n / 2} / \Gamma(n / 2)$ is the solid angle in $n$ dimensional Euclidean space subtended by the complete $(n-1)$-dimensional spherical surface and $\Gamma$ is the Gamma function. This gives the exact result of $4 \pi$ steradians for $n=3$. In general, the summation interpolant (9) refers to $\mathcal{N}$ non-uniformly distributed points and therefore the volumes $\Delta V_{b}$ may not be the same for all particles. In almost all SPH applications, the particle volume $\Delta V_{b}$ is replaced by the ratio $m_{b} / \rho_{b}$, where $m_{b}$ and $\rho_{b}$ are the mass and density of particle $b$, respectively. For uniformly distributed particles, Fulk [41] derived error bounds for the SPH approximation (9) through the use of Taylor series expansions and proved the following:

Lemma 1 (Consistency for the SPH Approximation). Given a function, $f(\mathbf{x}) \in \mathcal{S}\left(\mathbb{R}^{3}\right)$, and given a kernel interpolation function, $W$, that is symmetric, positive-definite, normalized, and has compact support, the SPH approximation (9) is consistent with the identity operator, $\operatorname{If}(\mathbf{x})=f(\mathbf{x})$, under the uniform norm,

$$
\begin{aligned}
\|S f-I f\|_{\infty} & =\|K f-I f+S f-K f\|_{\infty} \\
& \leq\|K f-I f\|_{\infty}+\|S f-K f\|_{\infty},
\end{aligned}
$$

provided that $\Delta V_{b}$ is equal to $m_{b} / \rho_{b}$, where If $=f\left(\mathbf{x}_{a}\right)$ is the value of the exact function at $\mathbf{x}_{a}$, and $K f=\left\langle f\left(\mathbf{x}_{a}\right)\right\rangle$ and $S f=f_{a}$ denote the kernel and the SPH approximations of $f(\mathbf{x})$ at $\mathbf{x}_{a}$ defined by Eqs. (1) and (9), respectively.

Fulk proved that in the limit of vanishing inter-particle distances $\| S f-$ $K f \|_{\infty} \rightarrow 0$, while $\|K f-I f\|_{\infty} \rightarrow 0$ as $h \rightarrow 0$. Lemma 1 is valid in any dimension. Similar conclusions follow for the SPH approximation of the 
gradient of a function. However, a definition of consistency for the particle approximation based solely on the equivalence $\Delta V_{b} \rightarrow m_{b} / \rho_{b}$ is incomplete because in general the integral conditions (2) and (4) in discrete form are not satisfied exactly, i.e.,

$$
\begin{aligned}
& \mathbf{M}_{0}=\sum_{b=1}^{\mathcal{N}} \frac{m_{b}}{\rho_{b}} W_{a b} \neq 1 \\
& \mathbf{M}_{l}=\sum_{b=1}^{\mathcal{N}} \frac{m_{b}}{\rho_{b}}\left(\mathbf{x}_{b}-\mathbf{x}_{a}\right)^{l} W_{a b} \neq \mathbf{0}^{(l)}, \text { for } l=1,2, \ldots, m .
\end{aligned}
$$

The same is true for the discrete form of the integral relations (8), leading to complete loss of consistency due to the particle approximation. Considering the analogy between quasi-Monte Carlo and SPH particle estimates, Monaghan [42] first conjectured that for low-discrepancy (i.e., quasi-regular or quasi-random) sequences of particles, as is indeed the case in SPH simulations, the error carried by the particle approximation is $O\left((\ln \mathcal{N})^{n} / \mathcal{N}\right)$.

The complexity of error behavior in SPH has been highlighted by Quinlan et al. [15] and Vaughan et al. [16]. The former authors used the second EulerMacLaurin formula to estimate this error for one-dimensional regularly and irregularly distributed particles. They found that for regular distributions as $h \rightarrow 0$, while maintaining constant the ratio of particle spacing to smoothing length, $\Delta x / h$, the error decays as $h^{2}$ until a limiting discretization error is reached, which is independent of $h$. If $\Delta x \rightarrow 0$ while maintaining $h$ constant, the error decays at a rate which depends on the kernel smoothness. When particles are distributed non-uniformly, decreasing $h$ with constant $\Delta x / h$ results in discretization-limited errors at best. On the other hand, Vaughan et al. [16] showed that if $C^{0}$ consistency is not achieved the error is $O\left(f(\mathcal{N})\right.$ ), which does not converge with $h$, whereas if $C^{1}$ consistency is achieved the error goes as $O\left(h^{2} f(\mathcal{N})\right)$. They concluded that if $f(\mathcal{N}) \sim$ $(\ln \mathcal{N})^{n} / \mathcal{N}$, an analytical solution for the functional dependence of the total number of particles $N$ on $h$ cannot be obtained. However, recently Zhu et al. [19] derived the parameterizations $h \propto N^{-1 / \beta}$ and $\mathcal{N} \propto N^{1-3 / \beta}$ for $\beta \in[5,7]$ based on a balance between the kernel and the particle approximation errors. For $\beta=6$, this gives $h \propto N^{-1 / 6}$ and $\mathcal{N} \propto N^{1 / 2}$. They stated that these scaling relations comply with the joint limit $N \rightarrow \infty, h \rightarrow 0, \mathcal{N} \rightarrow \infty$ as a necessary condition to achieve full particle consistency [43]. However, the systematic increase of the number of neighbors $\mathcal{N}$ with the total number 
of particles $N$ demands changing the interpolation kernel to a compactly supported Wendland-type function [44], which, unlike traditional kernels, is free from the so-called pairing instability when working with large numbers of neighbors [45].

\section{SPH errors in one-space dimension}

For simplicity, first consider the analysis for a set of irregularly distributed particles on the real line. Let $\phi(x) \in \mathcal{S}(\mathbb{R})$ be a test function and $\hat{\phi}(j)=$ $\int_{\mathbb{R}} \phi(x) \exp (-i 2 \pi j x) d x$ its Fourier transform, where $\hat{\phi}$ also belongs to $\mathcal{S}(\mathbb{R})$. The distributional relation

$$
\sum_{b=-\infty}^{\infty} \phi(b)=\sum_{j=-\infty}^{\infty} \hat{\phi}(j)=\int_{\mathbb{R}} \phi(b) d b+2 \sum_{j=1}^{\infty} \int_{\mathbb{R}} \phi(b) \cos (2 \pi j b) d b,
$$

defines the Poisson summation formula [46], where in the leftmost sum $b \in \mathbb{Z}$, while in the integrals on the right side $b \in \mathbb{R}$. Here, the integer $b$ in the leftmost sum belongs to the space of particle labels and the integer $j$ belongs to the dual of the space of labels. Setting $\phi(b)=f_{b} W_{a b} \Delta x_{b}$, the leftmost summation becomes

$$
\sum_{b=-\infty}^{\infty} \phi(b) \rightarrow f_{a}=\sum_{b=1}^{\mathcal{N}} \frac{m_{b}}{\rho_{b}} f_{b} W_{a b}
$$

for any $\mathcal{N} \in \mathbb{N}$. The sum on the left side of relation (14) is over $\mathbb{Z}$, while the one on the right side runs over the set $[1, \mathcal{N}]$, which is a subset of both $\mathbb{N}$ and $\mathbb{Z}$. Since the kernel $W_{a b}$ has compact support centered at the position of particle $a$, only the $\mathcal{N}$ points within the support of $W_{a b}$ will actually contribute to the sum on the right side of Eq. (14). Now setting $\phi(b)=$ $\left(m_{b} / \rho_{b}\right) f\left(x_{b}\right) W\left(\left|x_{a}-x_{b}\right|, h\right)$ in the integrals on the right side of Eq. (13), the Poisson summation formula becomes

$$
\begin{aligned}
f_{a} & =\int_{\Omega_{1}} f\left(x_{b}\right) W\left(\left|x_{a}-x_{b}\right|, h\right) \frac{m_{b}}{\rho_{b}} d b \\
& +2 \sum_{j=1}^{\infty} \int_{\Omega_{1}} f\left(x_{b}\right) W\left(\left|x_{a}-x_{b}\right|, h\right) \cos (2 \pi j b) \frac{m_{b}}{\rho_{b}} d b
\end{aligned}
$$

where $\Omega_{1} \in \operatorname{supp}(W)=\left[x_{a}-k h, x_{a}+k h\right]$. The first integral on the right side of Eq. (15) is the kernel approximation of $f(x)$ at point $x=x_{a}$ provided 
that the equivalence holds

$$
d x_{b}=\frac{m_{b}}{\rho_{b}} d b,
$$

which relates the position of a particle to its label. Integration of relation (16) over the interval $\left[x_{a}-k h, x_{a}+k h\right]$ yields

$$
b\left(x_{a}+k h\right)-b\left(x_{a}-k h\right)=\int_{x_{a}-k h}^{x_{a}+k h} \frac{\rho(x)}{m(x)} d x,
$$

where $m_{b}=m\left(x_{b}\right)$ and $\rho_{b}=\rho\left(x_{b}\right)$. Note that the above relation stands for non-uniformly spaced particles. For a set of equidistant points with spacing $\Delta$, Eq. (17) reduces to $x_{b}=b \Delta$ and the Poisson formula for a uniform distribution is recovered. Since there is a one-to-one correspondence between the particle position $x_{b}$ and its label $b$, the function $x_{b}=x(b)$ is bijective.

Expanding $f\left(x_{b}\right)$ in Taylor series about $x_{a}$ and inserting the result in Eq. (15) yields the difference $\mathcal{E}_{S}$ between the value of the exact function $f\left(x_{a}\right)$ and its particle approximation $f_{a}$, i.e.,

$$
\begin{aligned}
\mathcal{E}_{S} & =f_{a}-f\left(x_{a}\right)=\sum_{l=1}^{\infty} \frac{f^{(l)}\left(x_{a}\right)}{l !} \int_{\Omega_{1}}\left(x_{b}-x_{a}\right)^{l} W\left(\left|x_{a}-x_{b}\right|, h\right) d x_{b} \\
& +2 \sum_{j=1}^{\infty} \sum_{l=0}^{\infty} \frac{f^{(l)}\left(x_{a}\right)}{l !} \int_{\Omega_{1}}\left(x_{b}-x_{a}\right)^{l} W\left(\left|x_{a}-x_{b}\right|, h\right) \cos (2 \pi j b) d x_{b},
\end{aligned}
$$

where $f^{(l)}\left(x_{a}\right)$ is the $l$ th derivative of $f(x)$ evaluated at $x_{a}$. According to expansion (3), the first sum on the right side of Eq. (18) is the difference $\mathcal{E}_{K}=\left\langle f\left(x_{a}\right)\right\rangle-f\left(x_{a}\right)$ between the exact function and its kernel estimate, while the second sum is the deviation of the particle approximation from the kernel estimate $\mathcal{E}_{S K}=f_{a}-\left\langle f\left(x_{a}\right)\right\rangle$. The first new result from inspection of Eq. (18) is that the particle approximation contributes with error terms that are proportional to the cosine Fourier transform of the integral consistency relations (4). Since the kernel is a symmetric function, only those terms with $l$ even will contribute to the error. Relation (18) is all we need to establish the correct consistency constraints for both the kernel and the particle approximations.

The number of neighbors of particle $a$ within the kernel support can be defined by the floor function

$$
\mathcal{N}\left(x_{a}, h\right)=\left[b\left(x_{a}+k h\right)-b\left(x_{a}-k h\right)\right]+\eta,
$$


where $\eta=1$ if $x_{a}$ is an interpolation point and $\eta=0$ otherwise. From Eq. (17), the above definition is equivalent to

$$
\mathcal{N}\left(x_{a}, h\right)=\int_{x_{a}-k h}^{x_{a}+k h} \frac{\rho(x)}{m(x)} d x .
$$

It is easy to show that for small $h, \mathcal{N}\left(x_{a}, h\right)=2\left(d b / d x_{a}\right) k h+O\left(h^{3}\right)$. Using relation (17), this implies that

$$
\mathcal{N}\left(x_{a}, h\right)=2 \frac{\rho\left(x_{a}\right)}{m\left(x_{a}\right)} k h+O\left(h^{3}\right) .
$$

Since a necessary condition to achieve full particle consistency is that $\mathcal{N}\left(x_{a}, h\right) \rightarrow$ $\infty$ as $h \rightarrow 0$ and $N \rightarrow \infty$ [19], satisfaction of this joint limit demands that $\rho\left(x_{a}\right) / m\left(x_{a}\right) \sim h^{-\beta}$ (with $\beta>1$ ) in Eq. (21). In the continuous limit the density is an intensive physical variable and therefore the above scaling must translate into the requirement that the particle mass scales with $h$ as $h^{\beta}$ (with $\beta>1$ ) in order to ensure that $\mathcal{N}\left(x_{a}, h\right) \rightarrow \infty$ as $h \rightarrow 0$ in Eq. (21). This implies the additional important limit $m \rightarrow 0$ as $h \rightarrow 0$ as a further condition for consistency of the particle approximation. From the above scaling for the particle mass it follows that $\mathcal{N} \propto h^{1-\beta}$, which is the one-dimensional equivalent of the scaling $\mathcal{N} \propto h^{3-\beta}$ (with $\beta>3$ ) suggested by Zhu et al. [19] in three dimensions.

The double summation in Eq. (18) represents the discretization errors implied by the particle approximation and is a measure of its deviation from the kernel estimate: $\mathcal{E}_{S K}=f_{a}-\left\langle f\left(x_{a}\right)\right\rangle$. For any infinitely differentiable function $f(x) \in \mathcal{S}(\mathbb{R})$, the limit $\mathcal{E}_{S K} \rightarrow 0$ is achieved only if

$$
\begin{aligned}
M_{l}^{F} & =\sum_{j=1}^{\infty} \int_{\Omega_{1}}\left(x_{b}-x_{a}\right)^{l} W\left(\left|x_{a}-x_{b}\right|, h\right) \cos (2 \pi j b) d x_{b} \\
& =\lim _{\mathcal{N} \rightarrow \infty} \sum_{j=1}^{\mathcal{N}} \int_{\Omega_{1}}\left(x_{b}-x_{a}\right)^{l} W\left(\left|x_{a}-x_{b}\right|, h\right) \cos (2 \pi j b) d x_{b} \\
& =0,
\end{aligned}
$$

$\forall l$ even with $l \geq 0$. These relations represent particle consistency conditions. Note that in the intermediate equality of Eq. (22) we have used the regularization criterion (B.11) in Appendix B. In actual simulations the contribution 
of these integrals can be neglected only if $\cos (2 \pi j b)$ oscillates very rapidly within $\operatorname{supp}(W)=\left[x_{a}-k h, x_{a}+k h\right]$, i.e., when

$$
\left[b\left(x_{a}+k h\right)-b\left(x_{a}-k h\right)\right]+\eta=\mathcal{N}\left(x_{a}, h\right)>\frac{1}{j},
$$

which implies $\mathcal{N}\left(x_{a}, h\right)>1$ for $j=1$. Note that if inequality (23) holds for $j=1$, it will also hold for any $j \geq 2$.

\subsection{Error bounds}

The error of the kernel approximation of $f(x)$ at the position of particle $a$ is given by the first summation in Eq. (18). Bounds on this error have been previously derived by Fulk [41]. However, a derivation is repeated in Appendix A under the uniform norm

$$
\|K f-I f\|_{\infty}=\left\|\mathcal{E}_{K}\right\|_{\infty}=\sup _{x_{b} \in \Omega_{1}}\left|\mathcal{E}_{K}\right|
$$

by retaining only second-order terms in the summation. The result is

$$
\left|\mathcal{E}_{K}\right| \leq e_{r}^{(2)} h^{2}
$$

which implies second-order accuracy for the kernel approximation. A higher order error is also possible if a kernel that has higher order vanishing even moments is used.

The second summation in Eq. (18) gives the error of the particle relative to the kernel approximation. As for the kernel approximation, bounds on this error are also derived under the uniform norm

$$
\|S f-K f\|_{\infty}=\left\|\mathcal{E}_{K S}\right\|_{\infty}=\sup _{x_{b} \in \Omega_{1}}\left|\mathcal{E}_{K S}\right| .
$$

The result of this analysis is

$$
\left|\mathcal{E}_{K S}\right| \leq \frac{4}{\pi} a_{0} k \sum_{l=0}^{\infty} h^{l} \tilde{e}_{r}^{(l)}\left(\lim _{\mathcal{N} \rightarrow \infty} \frac{1}{\mathcal{N}} \sum_{j=1}^{\mathcal{N}} \frac{1}{j}\right)
$$

where $a_{0}>0$ is an upper bound for the kernel function and $\tilde{e}_{r}^{(l)}$ is defined in Appendix B, where the intermediate steps leading to inequality (27) are described. A bound for the term between parenthesis can be obtained using 
the following theorem for the estimation of the Euler-Mascheroni constant $\gamma=0.5572 \ldots$ [47]:

Theorem 1 For every natural number $\mathcal{N}$,

$$
\frac{1}{2(\mathcal{N}+1)}<\sum_{j=1}^{\mathcal{N}} \frac{1}{j}-\ln \mathcal{N}-\gamma<\frac{1}{2 \mathcal{N}}
$$

The proof of this theorem is given by Young [47]. The upper bound is the first term of an asymptotic expansion which can be used to compute $\gamma$. Solving for the summation term (i.e., the $\mathcal{N}$ th harmonic number) in (28), dividing by $\mathcal{N}$, and applying the limit when $\mathcal{N} \rightarrow \infty$ gives

$$
\lim _{\mathcal{N} \rightarrow \infty} \frac{1}{\mathcal{N}} \sum_{j=1}^{\mathcal{N}} \frac{1}{j} \leq \lim _{\mathcal{N} \rightarrow \infty}\left[\frac{\gamma}{\mathcal{N}}+\frac{\ln \mathcal{N}}{\mathcal{N}}+\frac{1}{2 \mathcal{N}^{2}}\right]=0
$$

so that $\left|\mathcal{E}_{K S}\right| \rightarrow 0$ when $\mathcal{N} \rightarrow \infty$ and the particle approximation converges to the kernel approximation. We note that the logarithmic term on the right side of the above inequality provides the dominant error for finite $\mathcal{N}$. This term is just the one-dimensional equivalent of the theoretical upper bound of the quasi-Monte Carlo method for low-discrepancy (quasi-random) sets of points. Since the limit when $\mathcal{N} \rightarrow \infty$ of $\ln \mathcal{N} / \mathcal{N}$ is equal to the limit when $\mathcal{N} \rightarrow \infty$ of $1 / \mathcal{N}$, we have the asymptotic expansion

$$
\lim _{\mathcal{N} \rightarrow \infty} \frac{1}{\mathcal{N}} \sum_{j=1}^{\mathcal{N}} \frac{1}{j} \leq \frac{(1+\gamma)}{\mathcal{N}}+O\left(\frac{1}{\mathcal{N}^{2}}\right)
$$

for $\mathcal{N} \rightarrow \infty$. Using this result into Eq. (27) and retaining terms up to $l=2$ in the expansion yields

$$
\left|\mathcal{E}_{K S}\right| \leq \frac{4(1+\gamma) a_{0} k}{\pi \mathcal{N}}\left(\tilde{e}_{r}^{(0)}+h \tilde{e}_{r}^{(1)}+h^{2} \tilde{e}_{r}^{(2)}\right)
$$

This shows that in the limit $\mathcal{N} \rightarrow \infty$, the particle discretization error vanishes $\left(\mathcal{E}_{K S} \rightarrow 0\right)$ and so $f_{a} \rightarrow\left\langle f\left(x_{a}\right)\right\rangle$, i.e., the particle estimate of the function approaches the kernel estimate independently of $h$. In inequality (31) the leading term is $\propto 1 / \mathcal{N}$, which gives a zeroth-order convergence rate even though $h \rightarrow 0$. 
From inequalities (10), (25), and (31) it follows that the error bound for the full SPH approximation under the uniform norm is

$$
\begin{aligned}
\|S f-I f\|_{\infty} & \leq\left|\mathcal{E}_{K}\right|+\left|\mathcal{E}_{K S}\right| \\
& \leq \frac{4(1+\gamma) a_{0} k}{\pi \mathcal{N}}\left(\tilde{e}_{r}^{(0)}+h \tilde{e}_{r}^{(1)}+h^{2} \tilde{e}_{r}^{(2)}\right)+h^{2} e_{r}^{(2)},
\end{aligned}
$$

which expresses the important result that complete consistency for the SPH estimate of a function can be guaranteed only when $\mathcal{N} \rightarrow \infty$ and $h \rightarrow 0$ provided that $N \rightarrow \infty$ and $m \rightarrow 0$. As a further remark, note that the scalings $m \propto h^{\beta}$ and $\mathcal{N} \propto h^{1-\beta}$ imply that $m \propto \mathcal{N}^{\beta /(1-\beta)}$. Since $\beta>1$, this means that by increasing $\mathcal{N}$ mass resolution is also improved. As an exercise, in Appendix C we apply the present method to Monaghan's [3] one-dimensional SPH convergence analysis for a linear function defined over an infinite set of equidistant particles.

\section{SPH errors in $n$-dimensional space}

Let $\Lambda \subset \mathbb{R}^{n}$ be a crystalline lattice and $\Phi(\mathbf{x}): \mathbb{R}^{n} \rightarrow \mathbb{R}$ a smooth function of locally finite support $\Gamma$ belonging to $\mathcal{D}\left(\mathbb{R}^{n}\right)$. The distributional Fourier transform of $\Phi$, namely $\hat{\Phi}$, in the dual lattice $\Lambda^{\star}$ is given by the $n$-dimensional Poisson's formula [46]

$$
\sum_{b_{1}, b_{2}, \ldots, b_{n} \in \Lambda} \Phi\left(b_{1}, b_{2}, \ldots, b_{n}\right)=\sum_{\mathbf{j} \in \Lambda^{\star}} \hat{\Phi}(\mathbf{j}),
$$

where the $n$-plet of integers $\left(b_{1}, b_{2}, \ldots, b_{n}\right)$, with $b_{i} \in \mathbb{Z}(i=1,2, \ldots, n)$, denotes the projections of the lattice node (or particle) labels $b \in \mathbb{Z}^{n}$ on the axes of an $n$-dimensional Cartesian coordinate system and $\mathbf{j}=\left(j_{1}, j_{2}, \ldots, j_{n}\right)$. Setting the summation on the left side of Eq. (33) equal to the summation on the right of Eq. (9) for the SPH approximation of a function $f(\mathbf{x}): \mathbb{R}^{n} \rightarrow \mathbb{R}$ at particle position $\mathbf{x}_{a}$, Poisson's formula becomes

$$
f_{a}=\sum_{\mathbf{j} \in \Lambda^{\star}} \int_{\Omega_{n}} f\left(\mathbf{x}_{b}\right) W\left(\left\|\mathbf{x}_{a}-\mathbf{x}_{b}\right\|, h\right) \exp (-i 2 \pi \mathbf{j} \cdot \mathbf{b}) \frac{m_{b}}{\rho_{b}} d^{n} \mathbf{b},
$$

where now $\mathbf{b} \in \mathbb{R}^{n}$ and $\Omega_{n} \in \operatorname{supp}(W)$ is the integration domain in $n$ dimensional Euclidean space. Since $\mathbf{b}=\mathbf{b}\left(\mathbf{x}_{b}\right)$ is bijective, it admits the 
inverse $\mathbf{x}_{b}=\mathbf{x}_{b}(\mathbf{b})$, which in differential form becomes $d^{n} \mathbf{x}_{b}=\left|\mathbf{J}_{\mathbf{x}_{b}}(\mathbf{b})\right| d^{n} \mathbf{b}$, where $\mathbf{J}_{\mathbf{x}_{b}}(\mathbf{b})$ is the Jacobian matrix of the transformation and

$$
\left|\mathbf{J}_{\mathbf{x}_{b}}(\mathbf{b})\right|=\left|\frac{\partial\left(x_{b_{1}}, x_{b_{2}}, \ldots, x_{b_{n}}\right)}{\partial\left(b_{1}, b_{2}, \ldots, b_{n}\right)}\right|=\frac{m_{b}}{\rho_{b}},
$$

is its determinant. This is the generalization of the differential form (16) in multiple dimensions.

Expanding $f\left(\mathbf{x}_{b}\right)$ in Taylor series about $\mathbf{x}_{a}$ and inserting the result in Eq. (34) yields the error between the particle approximation $f_{a}$ and the exact value of the function at the position of particle $a$

$$
\begin{aligned}
\mathcal{E}_{S}^{(n)} & =f_{a}-f\left(\mathbf{x}_{a}\right) \\
& =\sum_{l=1}^{\infty} \frac{1}{l !} \nabla^{(l)} f\left(\mathbf{x}_{a}\right):: \cdots: \int_{\Omega_{n}}\left(\mathbf{x}_{b}-\mathbf{x}_{a}\right)^{l} W\left(\left\|\mathbf{x}_{a}-\mathbf{x}_{b}\right\|, h\right) d^{n} \mathbf{x}_{b} \\
& +\sum_{\substack{\mathbf{j} \in \Lambda^{*} \\
\mathbf{j} \neq \mathbf{0}}}^{\infty} \sum_{l=0}^{\infty} \frac{1}{l !} \nabla^{(l)} f\left(\mathbf{x}_{a}\right):: \cdots: \mathbf{M}_{l}^{F}(\mathbf{j}),
\end{aligned}
$$

where $\mathbf{0}$ is the $n$-dimensional null vector and

$$
\mathbf{M}_{l}^{F}(\mathbf{j})=\int_{\Omega_{n}}\left(\mathbf{x}_{b}-\mathbf{x}_{a}\right)^{l} W\left(\left\|\mathbf{x}_{a}-\mathbf{x}_{b}\right\|, h\right) \exp (-i 2 \pi \mathbf{j} \cdot \mathbf{b}) d^{n} \mathbf{x}_{b} .
$$

The error carried by the particle approximation vanishes provided that $\mathbf{M}_{l}^{F}(\mathbf{j})=$ $\mathbf{0}^{(l)}$ for all values of $l$, which is the generalization in $n$-dimensions of the particle consistency relations (22).

The number of neighbors of particle $a$ within the spherical support of the kernel is therefore defined by

$$
\mathcal{N}\left(\mathbf{x}_{a}, h\right)=\int_{\Omega_{n}} \frac{\rho(\mathbf{x})}{m(\mathbf{x}, h)} d^{n} \mathbf{x},
$$

which for $h \ll 1$ becomes

$$
\mathcal{N}\left(\mathbf{x}_{a}, h\right)=\frac{B_{n}}{n} \frac{\rho\left(\mathbf{x}_{a}\right)}{m\left(\mathbf{x}_{a}, h\right)} k^{n} h^{n}+O\left(h^{n+2}\right) .
$$

Note that for $n=1, B_{n}=B_{1}=2$ and the asymptotic form (39) reduces to the one-dimensional expression (21). From Eq. (39) it follows that the 
limit $\mathcal{N} \rightarrow \infty$ as $h \rightarrow 0$ is satisfied only if the particle mass scales with $h$ as $h^{\beta}$, with $\beta>n$. This reproduces the scaling $\mathcal{N} \propto h^{3-\beta}$ for $n=3$ as was suggested by Zhu et al. [19]. Therefore, in $n$-dimensional space, the scaling relations $m \propto h^{\beta}$ and $\mathcal{N} \propto h^{n-\beta}$ are necessary conditions to guarantee complete particle consistency in the limit $h \rightarrow 0$. There is a subtle point behind this scaling: as the volume of the kernel support collapses to a point with no size at all when $h \rightarrow 0$, the mass within the support must also tends to zero in the limit to yield a finite density at that point. In this limit $\mathcal{N} / N \rightarrow \mathcal{V}_{n} / V$ as $N \rightarrow \infty$, where $V$ is the finite volume of the system. Since $N \rightarrow V \mathcal{N} / \mathcal{V}_{n} \sim h^{-\beta}, N \rightarrow \infty$ faster than $\mathcal{N}$ as $h \rightarrow 0$, i.e., $\mathcal{N} / N \rightarrow 0$ in the transition from the discrete to the continuous space. This last limit was first noted by Rasio [43] through a simple linear analysis of sound wave propagation in one dimension. The above scalings have implications on the minimum resolvable mass, $M_{\min }=\mathcal{N} m$, i.e., the mass within the kernel support. Since $m \propto h^{\beta}$ and $\mathcal{N} \propto h^{n-\beta}$, this implies that $M_{\min } \propto \mathcal{N}^{n /(n-\beta)}$. In three-space dimensions $(n=3), \beta$ varies between $\beta=5$ for quasi-ordered particle distributions and $\beta=7$ for random distributions [19]. With the intermediate choice of $\beta=6$, the minimum resolvable mass scales with $\mathcal{N}$ as $M_{\text {min }} \propto \mathcal{N}^{-1}$, implying that as $\mathcal{N}$ is increased mass resolution is effectively improved.

A further parameter that characterizes the SPH interpolation procedure is the distance between pairs of particles $\Delta\left(\mathbf{x}_{i}, \mathbf{x}_{j}\right)$, which provides a measure of their actual distribution within the support of the kernel. If there exist $\mathcal{N}(\mathbf{x}, h)$ particles within $\Omega_{n} \in \operatorname{supp}(W)$, then there will be $\mathcal{N}(\mathbf{x}, h)[\mathcal{N}(\mathbf{x}, h)-$ 1]/2 different distances $\Delta\left(\mathbf{x}_{i}, \mathbf{x}_{j}\right)$ between particle pairs, which for an irregularly distributed set will be bounded as

$$
\Delta_{\min } \leq \Delta\left(\mathbf{x}_{i}, \mathbf{x}_{j}\right) \leq \Delta_{\max }
$$

where $\Delta_{\min }$ and $\Delta_{\max }$ are, respectively, the minimum and maximum distances. The mean distance $\Delta_{\mathrm{m}}$ is given by

$$
\Delta_{\mathrm{m}}=\left[\frac{\mathcal{V}_{n}}{\mathcal{N}(\mathbf{x}, h)}\right]^{1 / n},
$$

where $\mathcal{V}_{n}=B_{n}(k h)^{n} / n$ is the volume of the kernel support.

\subsection{Error Bounds}

Error bounds for the SPH interpolation in $n$-dimensional space can be determined under the uniform norm (10) in terms of the sum of the differ- 
ence between the kernel approximation of a function and its exact value, $\mathcal{E}_{K}^{(n)}$, given by the first summation in Eq. (36), and the difference between the kernel and the particle approximations, $\mathcal{E}_{K S}^{(n)}$, represented by the second summation.

The bound of $\mathcal{E}_{K}^{(n)}$ is derived in Appendix D for completeness and the result is given by the inequality

$$
\left|\mathcal{E}_{K}^{(n)}\right| \leq e_{r}^{(2, n)} h^{2}
$$

where the second-order accuracy is not affected by the dimension.

In order to derive a bound for $\mathcal{E}_{K S}^{(n)}$ let us assume for simplicity that the crystalline lattice $\Lambda$ is a cube in $n$-dimensions and that the particles within the cube are unevenly distributed in a low-discrepancy sequence. Although in actual SPH applications the computational domains can have a variety of shapes, the assumption of a cube does not entail a loss of generality. The dual lattice $\Lambda^{\star}$ is also an $n$-dimensional cube with finite spectrum [46]. As shown in Appendix E, the error bound for the particle approximation in $n$-dimensions has the form

$$
\left|\mathcal{E}_{K S}^{(n)}\right| \leq\left(\frac{2}{\pi}\right)^{n} \frac{a_{0} B_{n} k^{n}}{n} \sum_{l=0}^{\infty} h^{l} \tilde{e}_{r}^{(l, n)}\left(\lim _{\mathcal{N} \rightarrow \infty} \frac{1}{\mathcal{N}} \prod_{s=1}^{n} \sum_{j_{s}=1}^{\mathcal{N}_{s}} \frac{1}{j_{s}}\right)
$$

where $\mathcal{N}_{s}=\left[2 k h_{s} / \Delta_{s}\right], h_{s}$ and $\Delta_{s}$ are the projections of $h$ and $\Delta_{\mathrm{m}}$ on the $s$ th-axis of an $n$-dimensional Cartesian system, respectively, $j_{s}$ is the $s$ th component of the wave vector $\mathbf{j}$, and the notation "[p]" means the largest positive integer less or equal to $p$. For low-discrepancy sequences of sample points with $\mathcal{N} \gg 1$, there will always exist an $n$-dimensional Cartesian system over which the projected mean distances $\Delta_{s} \approx \Delta_{\mathrm{m}}$, and so from Eq. (41) it follows that $\mathcal{N}_{s} \approx\left(2 k h_{s} / \mathcal{V}_{n}^{1 / n}\right) \mathcal{N}^{1 / n}$. For $N \rightarrow \infty, h_{s} \rightarrow h \sim \mathcal{V}_{n}^{1 / n}$ and therefore $\mathcal{N}_{s} \rightarrow \infty$ as $\mathcal{N} \rightarrow \infty$. From inequality (28), we find that

$$
\sum_{j_{s}=1}^{\mathcal{N}_{s}} \frac{1}{j_{s}}<\gamma+\ln \mathcal{N}_{s}+\frac{1}{2 \mathcal{N}_{s}}
$$


Using this into Eq. (43) yields

$$
\begin{aligned}
\left|\mathcal{E}_{K S}^{(n)}\right| & \leq\left(\frac{2}{\pi}\right)^{n} \frac{a_{0} B_{n} k^{n}}{n} \sum_{l=0}^{\infty} h^{l} \tilde{e}_{r}^{(l, n)}\left[\lim _{\mathcal{N}, \mathcal{N}_{s} \rightarrow \infty} \frac{1}{\mathcal{N}} \prod_{s=1}^{n}\left(\gamma+\ln \mathcal{N}_{s}+\frac{1}{2 \mathcal{N}_{s}}\right)\right] \\
& =\left(\frac{2}{\pi}\right)^{n} \frac{a_{0} B_{n} k^{n}}{n} \sum_{l=0}^{\infty} h^{l} \tilde{e}_{r}^{(l, n)}\left[\lim _{\mathcal{N}, \mathcal{N}_{s} \rightarrow \infty} \frac{1}{\mathcal{N}}\left(\gamma+\ln \mathcal{N}_{s}+\frac{1}{2 \mathcal{N}_{s}}\right)^{n}\right]
\end{aligned}
$$

where

$$
\begin{aligned}
\frac{1}{\mathcal{N}}\left(\gamma+\ln \mathcal{N}_{s}+\frac{1}{2 \mathcal{N}_{s}}\right)^{n} & =\frac{\mathcal{N}_{s}^{n}}{\mathcal{N}}\left(\frac{\gamma}{\mathcal{N}_{s}}+\frac{\ln \mathcal{N}_{s}}{\mathcal{N}_{s}}+\frac{1}{2 \mathcal{N}_{s}^{2}}\right)^{n} \\
& =\frac{\mathcal{N}_{s}^{n}}{\mathcal{N}} \sum_{k=0}^{n} \frac{n !}{2^{k} k !(n-k) !}\left(\frac{\gamma}{\mathcal{N}_{s}}+\frac{\ln \mathcal{N}_{s}}{\mathcal{N}_{s}}\right)^{n-k} \frac{1}{\mathcal{N}_{s}^{2 k}}
\end{aligned}
$$

Noting that in the limit when $\mathcal{N}_{s} \rightarrow \infty$, the relation $\gamma / \mathcal{N}_{s}+\ln \mathcal{N}_{s} / \mathcal{N}_{s}=$ $(\gamma+1) / \mathcal{N}_{s}$ holds, we have that

$$
\begin{aligned}
\lim _{\mathcal{N}, \mathcal{N}_{s} \rightarrow \infty} \frac{1}{\mathcal{N}}\left(\gamma+\ln \mathcal{N}_{s}+\frac{1}{2 \mathcal{N}_{s}}\right)^{n} & =\lim _{\mathcal{N}, \mathcal{N}_{s} \rightarrow \infty} \frac{\mathcal{N}_{s}^{n}}{\mathcal{N}}\left(\frac{\gamma}{\mathcal{N}_{s}}+\frac{\ln \mathcal{N}_{s}}{\mathcal{N}_{s}}+\frac{1}{2 \mathcal{N}_{s}^{2}}\right)^{n} \\
& =\lim _{\substack{\mathcal{N}, \mathcal{N}_{s} \rightarrow \infty \\
\mathcal{N}}} \sum_{k=0}^{n} \frac{n !}{k !(n-k) !} \frac{(\gamma+1)^{n-k}}{2^{k} \mathcal{N}_{s}^{k}}
\end{aligned}
$$

For low-discrepancy sequences of particles $\mathcal{N}_{s} \propto \mathcal{N}^{1 / n}$ and therefore

$$
\lim _{\mathcal{N}, \mathcal{N}_{s} \rightarrow \infty} \frac{1}{\mathcal{N}} \sum_{k=0}^{n} \frac{n !}{k !(n-k) !} \frac{(\gamma+1)^{n-k}}{2^{k} \mathcal{N}_{s}^{k}} \sim \lim _{\mathcal{N} \rightarrow \infty}\left[\frac{(\gamma+1)^{n}}{\mathcal{N}}+O\left(\frac{1}{\mathcal{N}^{1+1 / n}}\right)\right]
$$

From the above relations it follows that $\left|\mathcal{E}_{K S}^{(n)}\right| \rightarrow 0$ when $\mathcal{N} \rightarrow \infty$. Hence, the asymptotic expansion

$$
\lim _{\mathcal{N} \rightarrow \infty} \frac{1}{\mathcal{N}} \prod_{s=1}^{n} \sum_{j_{s}=1}^{\mathcal{N}_{s}} \frac{1}{j_{s}} \leq \frac{(\gamma+1)^{n}}{\mathcal{N}}+O\left(\frac{1}{\mathcal{N}^{1+1 / n}}\right)
$$


holds for $\mathcal{N} \rightarrow \infty$. For $n=1$, Eq. (49) reduces to Eq. (30) with the asymptotic bound $O\left(1 / \mathcal{N}^{2}\right)$, while for $n=2$ the asymptotic bound goes as $O\left(1 / \mathcal{N}^{3 / 2}\right)$ and for $n=3$ as $O\left(1 / \mathcal{N}^{4 / 3}\right)$, implying that when $\mathcal{N} \rightarrow \infty$ the error $\left|\mathcal{E}_{K S}^{(n)}\right| \rightarrow 0$ more slowly as the dimension is increased. Therefore, the upper bound on the particle approximation error takes the form

$$
\left|\mathcal{E}_{K S}^{(n)}\right| \leq\left(\frac{2}{\pi}\right)^{n} \frac{(1+\gamma)^{n} a_{0} B_{n} k^{n}}{n \mathcal{N}} \sum_{l=0}^{\infty} h^{l} \tilde{e}_{r}^{(l, n)}
$$

Retaining only the first two terms in the above summation and adding the bound on $\mathcal{E}_{K}^{(n)}$, as given by inequality (42), the error bound for the full SPH estimate of a function is

$$
\begin{aligned}
\|S f-I f\|_{\infty} & \leq\left|\mathcal{E}_{K}^{(n)}\right|+\left|\mathcal{E}_{K S}^{(n)}\right| \\
& \leq\left(\frac{2}{\pi}\right)^{n} \frac{(1+\gamma)^{n} a_{0} B_{n} k^{n}}{n \mathcal{N}}\left(\tilde{e}_{r}^{(0, n)}+h \tilde{e}_{r}^{(1, n)}+h^{2} \tilde{e}_{r}^{(2, n)}\right) \\
& +h^{2} e_{r}^{(2, n)}
\end{aligned}
$$

which is the $n$-dimensional counterpart of the one-dimensional error bound (32). According to (51), the leading error for the particle approximation in multidimensions goes also as $1 / \mathcal{N}$.

Following steps similar to those described here for the function estimate it can be demonstrated that the error bounds for the SPH estimate of the gradient obeys a dependence on the SPH parameters similar to that given by inequality (51).

\section{Discussion}

Using the definition $\mathcal{N}_{s}=\left[2 k h_{s} / \Delta_{s}\right]$ together with Eq. (41), the error bound (45) can be written in the alternative form

$$
\left|\mathcal{E}_{K S}^{(n)}\right| \leq\left(\frac{2}{\pi}\right)^{n} a_{0} \sum_{l=0}^{\infty} h^{l-n} \tilde{e}_{r}^{(l, n)} \lim _{\Delta_{\mathrm{m}}, \Delta_{s} \rightarrow 0}\left[\Delta_{\mathrm{m}}\left(\gamma-\ln \Delta_{s}+\ln \left(2 k h_{s}\right)+\frac{\Delta_{s}}{4 k h_{s}}\right)\right]^{n} .
$$

For $\Delta_{\mathrm{m}} \approx \Delta_{s}$, the term $q=-\Delta_{\mathrm{m}} \ln \Delta_{s} \approx-\Delta_{\mathrm{m}} \ln \Delta_{\mathrm{m}}$ provides a measure of the loss of the continuous field information due to the SPH discretization. As long as $\Delta_{m} \rightarrow 0, q \rightarrow 0$ and the continuous information is recovered. This is the essence of the theorem of SPH convergence. On the other hand, the 
term $q_{R}=-\Delta_{\mathrm{m}} \ln \Delta_{s}$ is a relative measure of the loss of information since it involves the projections of the mean distance on straight lines. Since the process of projection works on the way of reducing the information, it is more convenient to use the SPH interpolation formula on either equidistant or lowdiscrepancy (i.e., quasi-random) sequences of sample points for which $\Delta_{s} \approx$ $\Delta_{\mathrm{m}}$ rather than on randomly disordered sequences where $\Delta_{s}<\Delta_{\mathrm{m}}$. This point is connected to the average case complexity of multivariate integration [48], where to derive the average case complexity an optimal choice of the sample points is needed in the computation of multivariate integrals.

\subsection{Approximate partition of unity}

The meaning of partition of unity in SPH is sometimes misunderstood in the literature. This concept is tightly related to relation (41) and therefore to the volume of the kernel support. For instance, noting that this relation can be written as $m_{a} / \rho_{a}=B_{n} k^{n} h^{n} /(n \mathcal{N})+O\left(h^{n+2}\right)$ and that the volume of the kernel support is $\mathcal{V}_{n}=B_{n} k^{n} h^{n} / n$, we find that $m_{a} / \rho_{a}=\mathcal{V}_{n} / \mathcal{N}+O\left(h^{n+2}\right)$. If according to Lemma 1 , we associate the ratio $m_{a} / \rho_{a}$ to the volume $\Delta V_{a}$ of particle $a$ and sum over all particle within the kernel support, we then recover the relation $\left(\sum_{b=1}^{\mathcal{N}} \Delta V_{b}\right) / \mathcal{V}_{n}=1+O\left(h^{n+2}\right)$, which implies that for finite sizes of the kernel support independently of whether $h$ is fixed or variable, partition of unity can be achieved only approximately to order $O\left(h^{n+2}\right)$. Exact partition of unity can be achieved only when $h \rightarrow 0$, which in turn will demand that $N \rightarrow \infty, m \rightarrow 0$, and $\mathcal{N} \rightarrow \infty$, with $\mathcal{N} / N \rightarrow 0$, for full consistency. If this requirement is fulfilled, the conditions $M_{0, a}=1$ and $\mathbf{M}_{0, a}^{\prime}=\mathbf{0}$ will be exactly satisfied. Moreover, Zhu et al. [19] quantified numerically the deviation from an exact partition of unity by evaluating the standard deviation measured in the distributions of $M_{0, a}$ as a function of $\mathcal{N}$ for a low-discrepancy set of particles, finding that $\sigma\left(M_{0}\right) \propto \mathcal{N}^{-1}$ as is indeed predicted by the error bound (50). Therefore, the inconsistency in the volume estimate, as measured by (11), declines as $\mathcal{N}$ is increased. As this limit is observed, it was shown numerically that the SPH estimates of the derivatives converge essentially at the same rate as the estimate of the function and the approximations become insensitive to particle disorder [40]. Moreover, as $h \rightarrow 0$ the inconsistency implied by the kernel truncation near a boundary is strongly mitigated and so no special boundary treatment of the interpolation scheme is needed except for the conditions demanded by the physics. 


\subsection{Numerical experiments}

As a numerical experiment, we consider the SPH reproducibility of the test functions

$$
f_{2 \mathrm{D}}(x, y)=\sin \pi x \sin \pi y,
$$

defined over the intervals $x \in[0,1]$ and $y \in[0,1]$, and

$$
f_{3 \mathrm{D}}(x, y, z)=\sin \pi x \sin \pi y \sin \pi z,
$$

defined over the intervals $x \in[0,1], y \in[0,1]$, and $z \in[0,1]$, using the standard interpolation formula (9) and the scaling relations $\mathcal{N} \propto N^{1 / 2}$ and $h \propto N^{-1 / 6}[19]$. Out of the family of possible scalings describing the dependence of $\mathcal{N}$ and $h$ on $N$, we allow $h$ to vary with $N$ as $h=N^{-1 / 6}$ to obtain the scaling relations $\mathcal{N} \approx 2.81 N^{0.675}$ and $h \approx 1.29 \mathcal{N}^{-0.247}$. The exponents of these scalings are slightly larger than the reference values of 0.5 and $-1 / 3$ and were chosen to accommodate larger numbers of neighbors within the kernel support at the price of having correspondingly larger values of $h$. A Wendland $\mathrm{C}^{4}$ function [45]

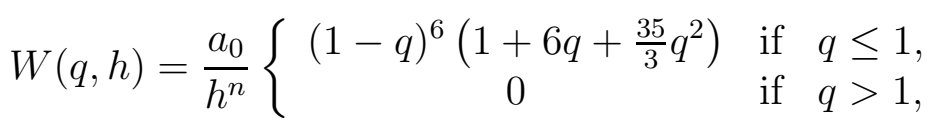

where $a_{0}=9 / \pi$ in $2 \mathrm{D}(n=2), a_{0}=495 /(32 \pi)$ in $3 \mathrm{D}(n=3)$, and $q=\left|\mathbf{x}-\mathbf{x}^{\prime}\right| / h$, is employed as the interpolation kernel. In the 2D case, the test function (53) is represented by filling the unit square plane with quasi-randomly distributed particles and varying the spatial resolution from $N=25^{2}$ to $N=1000^{2}$. The same irregular pattern is maintained for all resolutions. In the 3D case, the test function (54) is estimated by filling up the unit cube with particles placed in a similar quasi-random distribution and $N$ varying from $25^{3}$ to $200^{3}$ particles. The details of the resolution parameters employed in both sets of calculations are listed in Table 1 . In these analyses no boundary treatments are implemented at the borders of the computational domains.

For small values of $\mathcal{N}$ the smoothing length decreases rapidly as $\mathcal{N}$ increases and then more slowly at larger values of $\mathcal{N}$, asymptotically approaching zero as $\mathcal{N} \rightarrow \infty$. The error bound (51) for the test functions (53) and (54) reduces to

$$
\|S f-I f\|_{\infty} \leq \frac{36(1+\gamma)^{2}}{\pi^{2} \mathcal{N}}\left(1+2 \pi h+2 \pi^{2} h^{2}\right)+2 \pi^{2} h^{2}
$$


Table 1: Spatial resolution parameters

\begin{tabular}{rrrrrr}
\hline $2 \mathrm{D}$ & \multicolumn{5}{c}{$3 \mathrm{D}$} \\
\hline$N$ & $\mathcal{N}$ & \multicolumn{1}{c}{$h$} & \multicolumn{1}{c}{$N$} & \multicolumn{1}{c}{$\mathcal{L}$} & \multicolumn{1}{c}{$h$} \\
\hline $25^{2}$ & 216 & 0.342 & $25^{3}$ & 1903 & 0.200 \\
$50^{2}$ & 552 & 0.271 & $50^{3}$ & 7746 & 0.141 \\
$75^{2}$ & 955 & 0.237 & $75^{3}$ & 17607 & 0.115 \\
$100^{2}$ & 1408 & 0.215 & $100^{3}$ & 31528 & 0.100 \\
$125^{2}$ & 1903 & 0.200 & $125^{3}$ & 49539 & 0.089 \\
$150^{2}$ & 2434 & 0.188 & $150^{3}$ & 71662 & 0.082 \\
$175^{2}$ & 2998 & 0.179 & $175^{3}$ & 97917 & 0.075 \\
$200^{2}$ & 3590 & 0.171 & $200^{3}$ & 128319 & 0.071 \\
$250^{2}$ & 4852 & 0.159 & & & \\
$300^{2}$ & 6206 & 0.149 & & & \\
$400^{2}$ & 9151 & 0.136 & & & \\
$500^{2}$ & 12368 & 0.126 & & & \\
$750^{2}$ & 21381 & 0.110 & & & \\
$1000^{2}$ & 31528 & 0.100 & & & \\
\hline
\end{tabular}

and

$$
\|S f-I f\|_{\infty} \leq \frac{165(1+\gamma)^{3}}{\pi^{3} \mathcal{N}}\left(1+3 \pi h+\frac{9}{2} \pi^{2} h^{2}\right)+\frac{9}{2} \pi^{2} h^{2},
$$

respectively. Figure 1 shows the error bounds (solid circles and squares) along with the mean absolute errors (MAEs) between the SPH estimates of the test functions and their exact values (open circles and squares) calculated according to

$$
\mathcal{E}_{\mathrm{MAE}}=\frac{1}{N} \sum_{a=1}^{N}\left|S f_{a}-I f_{a}\right| .
$$

The MAEs and the error bounds (56) and (57) are plotted as a function of $N^{1 / 2}$ for the 2D runs (circles) and of $N^{1 / 3}$ for the 3D case (squares). This provides a better comparison of the errors with resolution in both dimensions. At comparable values of $N^{1 / 2}$ and $N^{1 / 3}$, the $3 \mathrm{D}$ particle distributions results in larger values of $\mathcal{N}$ and smaller smoothing lengths than the $2 \mathrm{D}$ case, and therefore the 3D error bounds are smaller than the 2D counterparts at all resolutions. As expected, the error bounds tend asymptotically to the MAEs as $N \rightarrow \infty$.

As it is well known, the volume estimate of a constant scalar field is not 
exactly represented by the standard SPH method because $M_{0} \neq 1$ [see Eq. (11)]. Note that the discrete form (11) represents the SPH estimate of a constant scalar field $f=1$. In order to measure the magnitude of this inconsistency and its dependence on $\mathcal{N}$, we calculate the distributions of $M_{0}$ using the parameters listed in Table 1 for our 2D and 3D irregularly distributed particles. The moment $M_{0}$ is expected to follow a peaked distribution around 1 with some errors for the discrete form (11) to approach the continuous normalization condition (2). Figures 2 and 3 display the distributions of $M_{0}$ for the $2 \mathrm{D}$ and $3 \mathrm{D}$ cases, respectively. For small values of $\mathcal{N}$ the distributions are broad and their maxima peak at values larger than 1 . This means that most particles fall within a narrow interval whose center deviates from unity and indicates some bias toward a density overestimate. However, the spread of the distributions is greatly reduced as $\mathcal{N}$ is increased. As this occurs, the inconsistency of the density estimates is reduced and the accuracy of the volume estimates increases in accordance with the $1 / \mathcal{N}$-dependence of the error bounds (56) and (57). As the experiment is repeated with even more neighbors, the distribution of $M_{0}$ approaches a Dirac- $\delta$ distribution with the error of the volume estimate decreasing to zero. When this limit is achieved, $C^{0}$-particle consistency of the SPH method is fully restored. We note that due to the symmetry of the kernel functions, $C^{1}$-particle consistency is automatically satisfied once $C^{0}$-consistency is achieved.

As a final remark, we may see that in most practical SPH applications the distribution of particles is unknown and therefore the overall quality of the density estimate is difficult to quantify. As was suggested by Zhu et al. [19], a simple procedure to measure the quality of the density estimate is just to calculate the distribution of $M_{0}$, and possibly all other higher moments up to the order of accuracy of the kernel function. A recent analysis of several SPH schemes has shown that in the limit of large $\mathcal{N}$ the estimates of a function and its first derivative converge to the same order independently of the degree of particle disorder [40]. Therefore, increasing $\mathcal{N}$ regulates the error observed in standard SPH when passing from a relaxed (regular or quasi-regular) distribution to an irregular (quasi-random) distribution. This has implications on relation (39) where exact partition of unity can be achieved only when $h \rightarrow 0$, which in turn demands that $N \rightarrow \infty, m \rightarrow 0$, and $\mathcal{N} \rightarrow \infty$. This is also related to the error carried by the approximation $\Delta V_{b} \approx m_{b} / \rho_{b}$, which is sensitive to the quality of the particle distribution. This error is also regulated by increasing $\mathcal{N}$ because as partition of unity is achieved the error of the approximation $\Delta V_{b} \approx m_{b} / \rho_{b}$ is correspondingly 
reduced as the volume of the kernel support $\mathcal{V}_{n} \rightarrow \sum_{b=1}^{\mathcal{N}} \Delta V_{b}$.

\subsection{Dependence of the error bounds on the kernel function}

In order to see how the type of the kernel function can influence the convergence rate, we repeat the experiments of previous section for four different kernel functions, namely the Lucy's quartic kernel [1]

$$
W(q, h)=\frac{a_{0}}{h^{n}}\left\{\begin{array}{cc}
(1-q)^{3}(1+3 q) & \text { if } \\
0 & \text { if } \quad q>1
\end{array}\right.
$$

where $a_{0}=5 / \pi$ for $n=2$ and $a_{0}=105 / 16 \pi$ for $n=3$, the Monaghan's super Gaussian kernel [49]

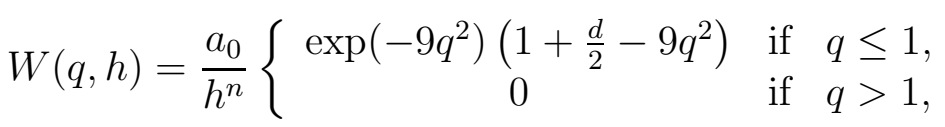

where $a_{0}=3^{n} / \pi^{n / 2}$, and the Wendland $\mathrm{C}^{2}$ and $\mathrm{C}^{6}$ functions given by

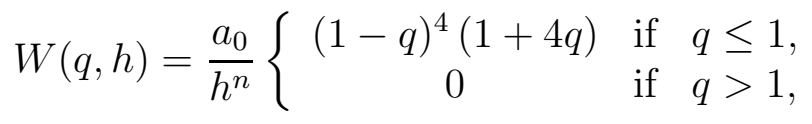

where $a_{0}=7 / \pi$ for $n=2$ and $a_{0}=21 /(2 \pi)$ for $n=3$, and

$$
W(q, h)=\frac{a_{0}}{h^{n}}\left\{\begin{array}{cl}
(1-q)^{8}\left(1+8 q+25 q^{2}+32 q^{3}\right) & \text { if } q \leq 1 \\
0 & \text { if } q>1
\end{array}\right.
$$

where $a_{0}=78 /(7 \pi)$ for $n=2$ and $a_{0}=1365 /(64 \pi)$ for $n=3$, respectively.

Using Eq. (10) the error bounds (56) and (57) for the test functions (53) and (54) can be written as

$$
\|S f-I f\|_{\infty}-\|K f-I f\|_{\infty} \leq\|S f-K f\|_{\infty}
$$

where

$$
\|S f-K f\|_{\infty} \leq \frac{A_{W}^{(2)}(1+\gamma)^{2}}{\pi^{2} \mathcal{N}}\left(1+2 \pi h+2 \pi^{2} h^{2}\right)
$$

and

$$
\|S f-K f\|_{\infty} \leq \frac{A_{W}^{(3)}(1+\gamma)^{3}}{\pi^{3} \mathcal{N}}\left(1+3 \pi h+\frac{9}{2} \pi^{2} h^{2}\right),
$$

are bounds for the difference between the particle and kernel approximations in $2 \mathrm{D}$ and $3 \mathrm{D}$, respectively. The parameters $A_{W}^{(n)}$ are numerical factors that 
depend on the kernel function. Thus, changing the kernel function will affect the convergence rate of the particle approximation only by a numerical factor. Table 2 lists the values of $A_{W}^{(n)}$ for the different kernel functions considered. According to these values, a better choice in $2 \mathrm{D}$ would be to use the Lucy's kernel, while in 3D the better choice would be to use the Wendland $\mathrm{C}^{2}$ function. However, as long as $\mathcal{N}$ increases, the differences between the various kernels become irrelevant since $\|S f-K f\|_{\infty} \rightarrow 0$ in the limit $\mathcal{N} \rightarrow \infty$ independently of the value of $h$. The mean absolute errors (58) obtained for the SPH estimates of the test functions (53) and (54) with the kernels (59)(62) and the same spatial resolution parameters of Table 1 follow the same trends of those plotted in Fig. 1 for the Wendland $\mathrm{C}^{4}$ kernel, with differences being less than about $2 \%$ at the lowest resolutions and less than $1 \%$ at the highest resolutions. Therefore, as the number of neighbors increases, the results become independent of the kernel function. However, when working with large numbers of neighbors we must take care of the fact that most conventional kernels suffer from a pairing instability, where particles come into close pairs and become less sensitive to small perturbations within the kernel support [45]. To overcome this difficulty, Wendland-type functions 44] are adopted, which have positive Fourier transforms and can support large numbers of neighbors without inducing a close pairing of particles [45].

\begin{tabular}{ccc}
\multicolumn{3}{c}{ Table 2: Numerical values of $A_{W}^{(n)}$} \\
\hline Kernel & $A_{2}^{(n)}$ & $A_{2}^{(n)}$ \\
\hline Lucy & 20 & 210 \\
Super Gaussian & 72 & $720 / \sqrt{\pi}$ \\
Wendland C & 28 & 112 \\
Wendland C $^{4}$ & 36 & 165 \\
Wendland C $^{6}$ & 156 & $455 / 2$ \\
\hline
\end{tabular}

According to the error bound (51), the accuracy of the particle approximation approaches that of the kernel approximation only when $\mathcal{N} \rightarrow \infty$ regardless of the value of $h$, while full convergence to the exact solution can be obtained only when also $h \rightarrow 0$. On the other hand, very small smoothing lengths with a small number of neighbors $(\mathcal{N}<100)$, as employed in most conventional SPH calculations, is not enough to guarantee convergence since in the limit $h \rightarrow 0$, an irreducible zeroth-order error term proportional to $1 / \mathcal{N}$ will still be present. 


\subsection{SPH approximation of the Dirac- $\delta$ distribution}

There is a point which has not been addressed directly in the SPH literature. This point is concerned with the SPH estimate of the Dirac- $\delta$ distribution. In principle, we may use the Poisson summation given by Eq. (15) to evaluate the convergence of the SPH estimate of the distribution $\delta\left(x_{a}-x_{0}\right)$, namely

$$
\begin{aligned}
\delta\left(x_{a}-x_{0}\right) & =\int_{\Omega_{1}} \delta\left(x_{b}-x_{0}\right) W\left(\left|x_{a}-x_{b}\right|, h\right) d x_{b} \\
& +2 \sum_{j=1}^{\infty} \int_{\Omega_{1}} \delta\left(x_{b}-x_{0}\right) W\left(\left|x_{a}-x_{b}\right|, h\right) \cos (2 \pi j b) d x_{b},
\end{aligned}
$$

where $x_{0} \in \mathbb{R}^{+}$. After using the sampling property of the Dirac- $\delta$ function, Eq. (66) reduces to

$$
\delta\left(x_{a}-x_{0}\right)=\left\{\begin{array}{ccc}
W\left(\left|x_{a}-x_{0}\right|, h\right)\left[1+2 \sum_{j=1}^{\infty} \cos \left(2 \pi j b_{0}\right)\right] & \text { if } & x_{0} \in \Omega_{1}, \\
0 & \text { if } & x_{0} \notin \Omega_{1},
\end{array}\right.
$$

where $b_{0} \in \mathbb{N}$ is a label associated to position $x_{0}$. Regularization of the

summation in Eq. (67) in the sense of the criterion (B.11) in Appendix B yields

$$
\sum_{j=1}^{\infty} \cos \left(2 \pi j b_{0}\right)=\lim _{\mathcal{N} \rightarrow \infty} \sum_{j=1}^{\mathcal{N}} \cos \left(2 \pi j b_{0}\right)=\lim _{\mathcal{N} \rightarrow \infty} \operatorname{Re}\left(\sum_{j=1}^{\mathcal{N}} \exp \left(i 2 \pi j b_{0}\right)\right),
$$

where $i=\sqrt{-1}$. If $b_{0} \in \mathbb{N}$, we have that

$$
\lim _{\mathcal{N} \rightarrow \infty} \operatorname{Re}\left(\sum_{j=1}^{\mathcal{N}} \exp \left(i 2 \pi j b_{0}\right)\right)=\infty
$$

while, if $b_{0} \in \mathbb{R} \backslash \mathbb{N}$, then any machine representation of $b_{0}$ is of the form $s+r / q$, with $s, r, q \in \mathbb{N}$ and $r<q$. Then,

$$
\operatorname{Re}\left(\sum_{j=1}^{\mathcal{N}} \exp \left(i 2 \pi j b_{0}\right)\right)=\operatorname{Re}\left\{\frac{[\exp (i 2 \pi \mathcal{N} r / q)-1]}{[\exp (i 2 \pi r / q)-1]} \exp (i 2 \pi r / q)\right\}=0,
$$

if $\mathcal{N} \equiv 0 \bmod q$. When $\mathcal{N} \not \equiv 0 \bmod q$, the series is different from zero and has a finite number of terms. This suggests that the SPH estimate of the Dirac- $\delta$ 
function has an oscillatory behavior as a function of $\mathcal{N}$. On the other hand, if the source pointed function is localized on a particle, the interpolation will diverge linearly from the exact solution even for $\mathcal{N} \gg 1$. To overcome these difficulties, the Dirac- $\delta$ distribution is replaced by a regularized smooth function

$$
\delta\left(x-x_{0}\right)=\lim _{\sigma \rightarrow 0^{+}} \eta_{\sigma}\left(x-x_{0}\right),
$$

where $\eta_{\sigma}(x)$ is sometimes called a nascent $\delta$ function, which has the following scaling properties

$$
\eta_{\sigma}(\mathbf{x})=\frac{1}{\sigma^{n}} \eta\left(\frac{\mathbf{x}}{\sigma}\right)
$$

where $\sigma$ is the bandwidth of $\eta_{\sigma}(x)$ and, as before, $n$ denotes the spatial dimension. For simplicity, let us consider a Gaussian distribution with standard deviation $\sigma$ so that

$$
\delta\left(x-x_{0}\right)=\lim _{\sigma \rightarrow 0^{+}} \frac{1}{\sqrt{\pi} \sigma} \exp \left[-\frac{\left(x-x_{0}\right)^{2}}{\sigma^{2}}\right],
$$

and perform the analysis in one-space dimension $(n=1)$. A similar procedure follows in two- and three-dimensions. We start by expanding an arbitrary function $f(x)$ in Taylor series about $x=x_{0}$ such that

$$
f(x)=f\left(x_{0}\right)+\sum_{k=1}^{\infty} \frac{1}{k !} f^{(k)}\left(x_{0}\right)\left(x-x_{0}\right)^{k} .
$$

According to the sampling property of the Dirac- $\delta$ distribution, we have that

$$
f\left(x_{0}\right)=\lim _{\sigma \rightarrow 0^{+}} \int_{-\infty}^{\infty} f(x) \frac{1}{\sqrt{\pi} \sigma} \exp \left[-\frac{\left(x-x_{0}\right)^{2}}{\sigma^{2}}\right] d x .
$$

Substitution of the expansion (74) into Eq. (75) gives for $f\left(x_{0}\right)$ the expression

$$
\begin{aligned}
f\left(x_{0}\right) & =\lim _{\sigma \rightarrow 0^{+}}\left\{f\left(x_{0}\right) \frac{1}{\sqrt{\pi} \sigma} \int_{-\infty}^{\infty} \exp \left[-\frac{\left(x-x_{0}\right)^{2}}{\sigma^{2}}\right] d x\right. \\
& +\frac{1}{\sqrt{\pi} \sigma} \sum_{k=0}^{\infty} \frac{1}{(2 k+1) !} f^{(2 k+1)}\left(x_{0}\right) \int_{-\infty}^{\infty}\left(x-x_{0}\right)^{2 k+1} \exp \left[-\frac{\left(x-x_{0}\right)^{2}}{\sigma^{2}}\right] d x \\
& \left.+\frac{1}{\sqrt{\pi} \sigma} \sum_{k=1}^{\infty} \frac{1}{(2 k) !} f^{(2 k)}\left(x_{0}\right) \int_{-\infty}^{\infty}\left(x-x_{0}\right)^{2 k} \exp \left[-\frac{\left(x-x_{0}\right)^{2}}{\sigma^{2}}\right] d x\right\}, \quad(76)
\end{aligned}
$$


where the first integral is equal to one by the normalization condition of the Gaussian distribution for $\forall \sigma \in \mathbb{R}^{+}$, the second term vanishes because the odd moments of the Gaussian distribution are exactly zero by symmetry, and the last term survives because the even moments obey the relation

$$
\int_{-\infty}^{\infty}\left(x-x_{0}\right)^{2 k} \exp \left[-\frac{\left(x-x_{0}\right)^{2}}{\sigma^{2}}\right] d x=\sigma^{2 k+1} \Gamma\left(k+\frac{1}{2}\right),
$$

where $\Gamma(z)$ is the Gamma function with $z>0$. Therefore,

$$
f\left(x_{0}\right)=f\left(x_{0}\right)+\lim _{\sigma \rightarrow 0^{+}} \frac{\sigma^{2 k}}{\sqrt{\pi}} \sum_{k=1}^{\infty} \frac{1}{(2 k) !} f^{(2 k)}\left(x_{0}\right) \Gamma\left(k+\frac{1}{2}\right),
$$

which demonstrates the validity of Eq. (71). Retaining only the first term in the summation, it is easy to see that in the limiting process to zero, the nascent Dirac- $\delta$ function is a second-order approximation to the Dirac- $\delta$ distribution since

$$
f\left(x_{0}\right)=f\left(x_{0}\right)+\lim _{\sigma \rightarrow 0^{+}}\left[\frac{1}{4} f^{(2)}\left(x_{0}\right) \sigma^{2}+O\left(\sigma^{4}\right)\right] .
$$

The same result is also obtained in $n$-dimensions where

$$
f\left(\mathbf{x}_{0}\right)=f\left(\mathbf{x}_{0}\right)+\lim _{\sigma \rightarrow 0^{+}}\left[\frac{1}{4} \nabla^{2} f\left(\mathbf{x}_{0}\right) \sigma^{2}+O\left(\sigma^{4}\right)\right] .
$$

As in Section 5.2, the error bound (51) for the SPH approximation of the Dirac- $\delta$ distribution in two-dimensions normalized to $1 /\left(\pi \sigma^{2}\right)$ can be calculated to give

$$
\|S f-I f\|_{\infty} \leq \frac{36(1+\gamma)^{2}}{\pi^{2} \mathcal{N}}\left(1+\frac{2 \sqrt{2}}{\sqrt{e}} \frac{h}{\sigma}+4 \frac{h^{2}}{\sigma^{2}}\right)+4 \frac{h^{2}}{\sigma^{2}}
$$

while after normalization by the factor $1 /\left(\pi^{3 / 2} \sigma^{3}\right)$, the error bound in threedimensions reads as follows

$$
\|S f-I f\|_{\infty} \leq \frac{165(1+\gamma)^{3}}{\pi^{3} \mathcal{N}}\left(1+3 \sqrt{2} \frac{h}{\sigma}+9 \frac{h^{2}}{\sigma^{2}}\right)+9 \frac{h^{2}}{\sigma^{2}} .
$$

From expressions (81) and (82) we may see immediately that regardless of the value of $h / \sigma$, the error due to the particle approximation decays as $\mathcal{N}^{-1}$, 
meaning that for large $\mathcal{N}$ this error is smaller than the kernel approximation error given by the last term in the right-hand side of expressions (81) and (82). Therefore, convergence to the Dirac- $\delta$ distribution can only be obtained if, in addition, $h \rightarrow 0$ faster than $\sigma$. Therefore, only in the limit when $\mathcal{N} \rightarrow \infty$, $h \rightarrow 0$, and $\sigma \rightarrow 0$, with $h / \sigma \rightarrow 0$, complete convergence is achieved. Since the number of particles within an $n$-dimensional sphere of radius $\sigma$ around the maximum of the Gaussian distribution is

$$
\mathcal{N}_{\sigma}=\left(\frac{\sigma}{k h}\right)^{n} \mathcal{N}
$$

we have that for $k=1$

$$
\frac{\mathcal{N}}{\mathcal{N}_{\sigma}}=\frac{h^{2}}{\sigma^{2}}
$$

for $n=2$, and

$$
\frac{\mathcal{N}}{\mathcal{N}_{\sigma}}=\frac{h^{3}}{\sigma^{3}}
$$

for $n=3$. Replacing these expressions in Eqs. (81) and (82), we may see that claiming that $h / \sigma \ll 1$ for strict convergence is equivalent to the requirement that $\mathcal{N} / \mathcal{N}_{\sigma} \ll 1$, i.e., the number of particles within a sphere of radius $\sigma$ must always be larger than the number of neighbors within the support of the interpolating kernel.

Table 3: Spatial resolution parameters for the SPH Dirac- $\delta$ estimation

\begin{tabular}{rrrrr}
\hline \multicolumn{5}{c}{$(h / \sigma)$} \\
\hline \multicolumn{1}{c}{$\mathcal{N}$} & $\mathcal{N}$ & $\sigma=0.1$ & $\sigma=0.01$ & $\sigma=0.001$ \\
\hline $2 \mathrm{D}$ & & & & \\
\hline $50^{2}$ & 552 & 2.71 & 27.1 & 271 \\
$125^{2}$ & 1903 & 2.00 & 20.0 & 200 \\
$200^{2}$ & 3590 & 1.71 & 17.1 & 171 \\
$400^{2}$ & 9151 & 1.36 & 13.6 & 136 \\
$1000^{2}$ & 31528 & 1.00 & 10.0 & 100 \\
\hline $3 \mathrm{D}$ & & & & \\
\hline $50^{3}$ & 7746 & 1.41 & 14.1 & 141 \\
$125^{3}$ & 49539 & 0.89 & 8.9 & 89 \\
$200^{3}$ & 128319 & 0.71 & 7.1 & 71 \\
\hline
\end{tabular}

Table 3 lists the values of $h / \sigma$ for the 2D and 3D experiments when $\sigma$ takes values of $0.1,0.01$, and 0.001 . For the $2 \mathrm{D}$ case, we may see that for all 
the resolutions tried in this paper $h / \sigma \geq 1$, implying that at these spatial resolutions convergence will never be achieved as was confirmed numerically for the case $f(x, y)=\delta\left(x-x_{0}\right) \delta\left(y-y_{0}\right)$, with $x_{0}=0.5$ and defined over the intervals $x \in[0,1]$ and $y \in[0,1]$. In the $3 \mathrm{D}$ case, for a function $f(x, y, z)=$ $\delta\left(x-x_{0}\right) \delta\left(y-y_{0}\right) \delta\left(z-z_{0}\right)$, with $x_{0}=0.5$ and defined over the intervals $x \in[0,1], y \in[0,1]$, and $z \in[0,1]$, only when $\sigma=0.1$ is the ratio $h / \sigma<1$ for the highest resolutions. However, at these resolutions as $\sigma \rightarrow 0$, the ratio $h / \sigma$ becomes larger and larger, implying that convergence to the Dirac$\delta$ distribution would demand increasing both $N$ and $\mathcal{N}$ such that in the limiting process $h / \sigma \rightarrow 0$. As expected the numerical simulations show that for the resolutions of Table 3 the MAEs oscillate as predicted by Eq. (67).

\subsection{Rounding error analysis of the SPH interpolation formula}

We now consider the error made when the particle approximation given by Eq. (9) is computed in floating-point. The summation (9) is an extended sequence of additions and products, where $f_{b}, W_{a b}$, and $\Delta V_{b}$ are standard floating-point numbers. In this sequence, the products are computed first and then added in the order in which they are written. The floating-point machine number that corresponds to the real value of the particle estimate $f_{a}$ is denoted by [50]

$$
\begin{aligned}
\mathrm{fl}\left(f_{a}\right) & =\mathrm{fl}\left(\sum_{b=1}^{\mathcal{N}} f_{b} W_{a b} \Delta V_{b}\right) \\
& =\mathrm{fl}\left(f_{1} W_{a 1} \Delta V_{1}+f_{2} W_{a 2} \Delta V_{2}+\cdots+f_{\mathcal{N}} W_{a \mathcal{N}} \Delta V_{\mathcal{N}}\right) .
\end{aligned}
$$

In order to evaluate Eq. (86) we must define the following quantities recursively

$$
\begin{aligned}
s_{1} & =\mathrm{fl}\left(f_{1} W_{a 1} \Delta V_{1}\right)=f_{1} W_{a 1} \Delta V_{1}\left(1+\eta_{1}\right), \\
s_{2} & =\mathrm{fl}\left(s_{1}+\mathrm{fl}\left(f_{2} W_{a 2} \Delta V_{2}\right)\right)=\mathrm{fl}\left(s_{1}+f_{2} W_{a 2} \Delta V_{2}\left(1+\eta_{2}\right)\right) \\
& =f_{1} W_{a 1} \Delta V_{1}\left(1+\eta_{1}\right)\left(1+\delta_{2}\right)+f_{1} W_{a 2} \Delta V_{2}\left(1+\eta_{2}\right)\left(1+\delta_{2}\right), \\
s_{3} & =\mathrm{fl}\left(s_{2}+\mathrm{fl}\left(f_{3} W_{a 3} \Delta V_{3}\right)\right)=\mathrm{fl}\left(s_{2}+f_{3} W_{a 3} \Delta V_{3}\left(1+\eta_{3}\right)\right) \\
& =f_{1} W_{a 1} \Delta V_{1}\left(1+\eta_{1}\right)\left(1+\delta_{2}\right)\left(1+\delta_{3}\right)+ \\
& +f_{2} W_{a 2} \Delta V_{2}\left(1+\eta_{2}\right)\left(1+\delta_{2}\right)\left(1+\delta_{3}\right)+ \\
& +f_{3} W_{a 3} \Delta V_{3}\left(1+\eta_{3}\right)\left(1+\delta_{3}\right),
\end{aligned}
$$


and so on. By induction, we have for $b=\mathcal{N}$ that

$$
\begin{aligned}
s_{\mathcal{N}} & =f_{1} W_{a 1} \Delta V_{1}\left(1+\eta_{1}\right)\left(1+\delta_{2}\right)\left(1+\delta_{3}\right)+\cdots+\left(1+\delta_{\mathcal{N}}\right)+ \\
& +f_{2} W_{a 2} \Delta V_{2}\left(1+\eta_{2}\right)\left(1+\delta_{2}\right)\left(1+\delta_{3}\right)+\cdots+\left(1+\delta_{\mathcal{N}}\right)+ \\
& +\cdots+f_{\mathcal{N}} W_{a \mathcal{N}} \Delta V_{\mathcal{N}}\left(1+\eta_{\mathcal{N}}\right)\left(1+\delta_{\mathcal{N}}\right),
\end{aligned}
$$

where $\left|\eta_{b}\right| \leq u$ and $\left|\delta_{b}\right| \leq u$, with $\delta_{1}=0$. Here, $u$ is the unit roundoff error defined as $u=\epsilon / 2$, where $\epsilon=\beta^{-(t-1)}$ is the so-called machine epsilon. In IEEE standard double precision, $\beta=2$ and $t=53$ so that $\epsilon=2^{-52}=2.220446 \times 10^{-16}$ and $u=1.110223 \times 10^{-16}$. From Eqs. (87) and (88) it follows that

$$
\mathrm{fl}\left(f_{a}\right)=\sum_{b=1}^{\mathcal{N}} f_{b} W_{a b} \Delta V_{b}\left(1+\eta_{b}\right) \prod_{c=b}^{\mathcal{N}}\left(1+\delta_{c}\right) .
$$

For $b=1$, the number of coefficients of $f_{1} W_{a 1} \Delta V_{1}$ is equal to $\mathcal{N}$ since $\delta_{1}=0$, while for $b>1$, the number of coefficients of $f_{b} W_{a b} \Delta V_{b}$ is just $\mathcal{N}-b+2$. Since $\left|\eta_{b}\right| \leq u$ and $\left|\delta_{c}\right| \leq u$, we may write that

$$
\left(1+\eta_{b}\right) \prod_{c=b}^{\mathcal{N}}\left(1+\delta_{c}\right) \approx\left(1+\eta_{b}\right)(1+\delta)^{\mathcal{N}-1} \approx(1+\delta)^{\mathcal{N}} .
$$

Now, expanding in Taylor series $(1+\delta)^{\mathcal{N}}$ about $\delta=0$ and retaining terms up to the first order, we have that

$$
(1+\delta)^{\mathcal{N}} \approx 1+\mathcal{N} \delta
$$

so that Eq. (89) can be written in the much simpler form

$$
\mathrm{fl}\left(f_{a}\right)=(1+\mathcal{N} \delta) \sum_{b=1}^{\mathcal{N}} f_{b} W_{a b} \Delta V_{b}=(1+\mathcal{N} \delta) f_{a},
$$

with $|\delta| \leq \epsilon / 2$. Therefore, a bound on the forward error involved in the operation is

$$
\begin{aligned}
\left|\mathrm{f}\left(\sum_{b=1}^{\mathcal{N}} f_{b} W_{a b} \Delta V_{b}\right)-\sum_{b=1}^{\mathcal{N}} f_{b} W_{a b} \Delta V_{b}\right| & \leq\left|\mathcal{N} \delta \sum_{b=1}^{\mathcal{N}} f_{b} W_{a b} \Delta V_{b}\right| \\
& \leq \mathcal{N}|\delta| \sum_{b=1}^{\mathcal{N}}\left|f_{b}\right| W_{a b} \Delta V_{b} \\
& \leq \frac{1}{2} \mathcal{N} \epsilon\left|f_{a}\right| .
\end{aligned}
$$


From the last inequality in Eq. (93) it follows that the particle estimate $f_{a}$ is approximated by a floating number $\mathrm{fl}\left(f_{a}\right)$ with a relative error no larger than $\mathcal{N} \epsilon / 2$. In IEEE standard double precision, this error is $\leq 1.110223 \times 10^{-16} \mathcal{N}$ and becomes larger with larger number of neighbors.

\section{Conclusions}

The consistency and convergence of the smoothed particle hydrodynamics (SPH) interpolation formula for the estimate of a function was investigated by analytical means. Because of the widespread use of SPH in science and engineering, the issue of SPH consistency has become a very hot and important topic of research. The method employed to derive the explicit functional dependence of the error bounds on the SPH interpolation parameters for the particle approximation of a function is based on the Poisson summation formula for kernels with a locally finite support. The results of the analysis not only clarify the issue of SPH consistency, but also permit assessing the accuracy of the standard SPH interpolation formula which has been thought to be a non-trivial problem.

The advantage of using the Poisson summation formula is that it enables the simultaneous treatment of both the kernel and particle approximation errors from which new consistency integral relations for the particle estimate follow as the cosine Fourier transform of the kernel consistency relations. The functional dependence of the error bounds on the SPH parameters, namely the smoothing length, $h$, and the number of neighbors, $\mathcal{N}$, within the kernel support is derived explicitly from which consistency conditions arise. In particular, as long as $\mathcal{N} \rightarrow \infty$, the particle approximation converges to the kernel approximation independently of $h$ provided that the particle mass scales with $h$ as $m \propto h^{\beta}$, with $\beta>n$, where $n$ is the spatial dimension. This implies that as $h \rightarrow 0$, the joint limit $m \rightarrow 0, \mathcal{N} \rightarrow \infty$, and $N \rightarrow \infty$ is necessary to restore complete consistency, where $N$ is the total number of particles. The requirement that $m \rightarrow 0$ as $h \rightarrow 0$ leads to the scaling $\mathcal{N} \propto$ $h^{n-\beta}$ [19]. In addition, for finite values of $\mathcal{N}$ a dominant error of the form $(\ln \mathcal{N})^{n} / \mathcal{N}$ emerges from the present analysis, as was first conjectured by Monaghan [42] based on the similarity between the SPH and the quasi-Monte Carlo estimates. For $\mathcal{N} \gg 1$, the present analysis predicts that the error of the SPH approximation declines as $\mathcal{N}^{-1}$ independently of the dimension, guaranteeing approximate partition of unity of the kernel volume. 
In the light of the above results, the Poisson summation formula appears to be a powerful tool for the error analysis of particle methods involving the evaluation of quadratures, as is also the case of the element-free Galerkin (EFG and GEFG), the reproducing kernel particle (RKPM and GRKPM), the moving least squares (MLSM and GMLSM), the Monte Carlo, and the quasi-Monte Carlo schemes among others. On the other hand, application of the present method to the analysis of the fluid-dynamics SPH equations will allow to formally assess the accuracy and convergence of current $\mathrm{SPH}$ simulations by analytical means.

\section{Appendices}

\section{A. Error bound for the kernel approximation in one dimension}

Using the kernel normalization condition (2) and recalling that the first moment $(l=1)$ of the kernel vanishes identically, the kernel approximation of $f(x)$ at the position of particle $a$ follows from the first summation on the right side of Eq. (18) as

$$
\begin{aligned}
\left\langle f\left(x_{a}\right)\right\rangle & =f\left(x_{a}\right) \\
& +\frac{1}{2} f^{(2)}\left(x_{a}\right) \int_{\Omega_{1}}\left(x_{b}-x_{a}\right)^{2} W\left(\left|x_{a}-x_{b}\right|, h\right) d x_{b},
\end{aligned}
$$

where only terms up to $l=2$ have been retained. Using the Cauchy-Schwarz inequality it follows that

$$
\begin{aligned}
\left|\mathcal{E}_{K}\right| & =\frac{1}{2}\left|f^{(2)}\left(x_{a}\right) \int_{\Omega_{1}}\left(x_{b}-x_{a}\right)^{2} W\left(\left|x_{a}-x_{b}\right|, h\right) d x_{b}\right| \\
& \leq \frac{1}{2}\left|f^{(2)}\left(x_{a}\right)\right| \int_{\Omega_{1}}\left|\left(x_{b}-x_{a}\right)^{2}\right| W\left(\left|x_{a}-x_{b}\right|, h\right) d x_{b} .
\end{aligned}
$$

Noting that $\left|\left(x_{b}-x_{a}\right)^{2}\right| \leq k^{2} h^{2}$ and defining

$$
e_{r}^{(2)}=\frac{k^{2}}{2} \sup _{\xi \in \Omega_{1}}\left|f^{(2)}(\xi)\right| \geq \frac{k^{2}}{2}\left|f^{(2)}(\xi)\right|,
$$

the bound is

$$
\left|\mathcal{E}_{K}\right| \leq e_{r}^{(2)} h^{2} \int_{\Omega_{1}} W\left(\left|x_{a}-x_{b}\right|, h\right) d x_{b}=e_{r}^{(2)} h^{2},
$$

which is second-order in $h$. 


\section{B. Error bound for the particle approximation in one dimension}

The double summation in the second term on the right side of Eq. (18) gives the error due to the particle discretization and represents the difference $\mathcal{E}_{K S}=f_{a}-\left\langle f\left(x_{a}\right)\right\rangle$ between the particle approximation and the kernel estimate of $f(x)$ evaluated at the position of particle $a$. The steps involved in the derivation of inequality (27) are described here starting from

$$
\mathcal{E}_{K S}=2 \sum_{j=1}^{\infty} \sum_{l=0}^{\infty} \frac{f^{(l)}\left(x_{a}\right)}{l !} \int_{\Omega_{1}}\left(x_{b}-x_{a}\right)^{l} W\left(\left|x_{a}-x_{b}\right|, h\right) \cos (2 \pi j b) d x_{b} .
$$

To obtain a bound on this error term first define

$$
\tilde{e}_{r}^{(l)}=\frac{k^{l}}{l !} \sup _{\xi \in \Omega_{1}}\left|f^{(l)}(\xi)\right| \geq \frac{k^{l}}{l !}\left|f^{(l)}(\xi)\right|,
$$

and $\left|\left(x_{b}-x_{a}\right)^{l}\right| \leq k^{l} h^{l}$. Moreover, since any suitable kernel function achieves a maximum value at the position of the observation point, i.e., $\max \left\{W \mid\left(x_{a}-\right.\right.$ $\left.\left.x_{b} \mid, h\right)\right\}=W(0, h)=a_{0} / h$ when $x_{b}=x_{a}$, it follows that $W\left(\left|x_{a}-x_{b}\right|, h\right) \leq$ $a_{0} / h$ for any $x_{b} \in \operatorname{supp}(W)$, where $a_{0}$ is a positive constant. Therefore,

$$
\begin{aligned}
\left|\mathcal{E}_{K S}\right| & =2\left|\sum_{j=1}^{\infty} \sum_{l=0}^{\infty} \frac{f^{(l)}\left(x_{a}\right)}{l !} \int_{\Omega_{1}}\left(x_{b}-x_{a}\right)^{l} W\left(\left|x_{a}-x_{b}\right|, h\right) \cos (2 \pi j b) d x_{b}\right| \\
& \leq 2 \sum_{j=1}^{\infty} \sum_{l=0}^{\infty} \frac{1}{l !}\left|f^{(l)}\left(x_{a}\right)\right|\left|\int_{\Omega_{1}}\left(x_{b}-x_{a}\right)^{l} W\left(\left|x_{a}-x_{b}\right|, h\right) \cos (2 \pi j b) d x_{b}\right| .
\end{aligned}
$$

Let $x_{b, \max }$ be a point within $\Omega_{1}$ such that $\left(x_{b, \max }-x_{a}\right)^{l} W\left(\left|x_{a}-x_{b, \max }\right|, h\right)=$ $\max \left\{\left(x_{b}-x_{a}\right)^{l} W\left(\left|x_{a}-x_{b}\right|, h\right)\right\}, \forall x_{b} \in \Omega_{1}$. Using the Cauchy-Schwarz inequality in (B.3), the error bound follows as

$$
\begin{aligned}
\left|\mathcal{E}_{K S}\right| & \leq 2 \sum_{j=1}^{\infty} \sum_{l=0}^{\infty} \frac{1}{l !}\left|f^{(l)}\left(x_{a}\right)\right|\left|\left(x_{b, \max }-x_{a}\right)^{l}\right| W\left(\left|x_{a}-x_{b, \max }\right|, h\right)\left|\int_{\Omega_{1}} \cos (2 \pi j b) d x_{b}\right| \\
& \leq 2 a_{0} \sum_{l=0}^{\infty} h^{l-1} \tilde{e}_{r}^{(l)} \sum_{j=1}^{\infty}\left|\int_{\Omega_{1}} \cos (2 \pi j b) d x_{b}\right|
\end{aligned}
$$


where relation (B.2) and the definitions $\left|\left(x_{b, \max }-x_{a}\right)^{l}\right| \leq k^{l} h^{l}$ and $W\left(\mid x_{a}-\right.$ $\left.x_{b, \max } \mid, h\right) \leq a_{0} / h$ have been used. To evaluate the cosine integral first expand $b=b\left(x_{b}\right)$ about $x_{a}$ to produce the linear mapping

$$
b\left(x_{b}\right)=b\left(x_{a}\right)+\left(\frac{d b}{d x_{b}}\right)_{x_{b}=x_{a}}\left(x_{b}-x_{a}\right)+O\left[\left(x_{b}-x_{a}\right)^{2}\right],
$$

where according to relations (16) and (21)

$$
\left(\frac{d b}{d x_{b}}\right)_{x_{b}=x_{a}}=\frac{\rho\left(x_{a}\right)}{m\left(x_{a}, h\right)}=\frac{\mathcal{N}\left(x_{a}, h\right)}{2 k h} .
$$

Note that if $\cos (2 \pi j b)$ varies rapidly within the domain $\Omega_{1}$ the above integral vanishes, which is a necessary requirement to test its convergence to zero when $\mathcal{N} \gg 1$. Replacing relation (B.6) into expansion (B.5), the cosine integral becomes

$$
\begin{aligned}
\int_{\Omega_{1}} \cos (2 \pi j b) d x_{b} & =\cos \left[2 \pi j b\left(x_{a}\right)\right] \int_{x_{a}-k h}^{x_{a}+k h} \cos \left[\frac{2 \pi}{P} x_{b a}\right] d x_{b} \\
& -\sin \left[2 \pi j b\left(x_{a}\right)\right] \int_{x_{a}-k h}^{x_{a}+k h} \sin \left[\frac{2 \pi}{P} x_{b a}\right] d x_{b}
\end{aligned}
$$

where $P=2 h k / j \mathcal{N}(\xi, h)$ and $x_{b a}=x_{b}-x_{a}$. The sine integral vanishes identically, while the cosine integral has a maximum value equal to $P / \pi$. Therefore,

$$
\left|\int_{x_{a}-k h}^{x_{a}+k h} \cos \left[\frac{2 \pi}{P} x_{b a}\right] d x_{b}\right| \leq \frac{P}{\pi}
$$

from which it follows that

$$
\left|\int_{\Omega_{1}} \cos (2 \pi j b) d x_{b}\right| \leq\left|\cos \left[2 \pi j b\left(x_{a}\right)\right]\right| \frac{2 k h}{\pi j \mathcal{N}\left(x_{a}, h\right)} \leq \frac{2 k h}{\pi j \mathcal{N}\left(x_{a}, h\right)} .
$$

Replacing this bound into (B.4) leads to inequality (27)

$$
\left|\mathcal{E}_{\mathcal{K S}}\right| \leq \frac{4}{\pi} a_{0} k \sum_{l=0}^{\infty} h^{l} \tilde{e}_{r}^{(l)}\left(\lim _{\mathcal{N}\left(x_{a}, h\right) \rightarrow \infty} \frac{1}{\mathcal{N}\left(x_{a}, h\right)} \sum_{j=1}^{\mathcal{N}\left(x_{a}, h\right)} \frac{1}{j}\right) .
$$

In writing inequality (B.10) the following must be noticed. First, returning to Eq. (13) we recall that the function $\phi(b)$ in the leftmost sum has locally finite 
support and therefore the correspondence in Eq. (14) holds, where the integer $b \in[1, \mathcal{N}]$. That is, only the $\mathcal{N}$ sample points within the support of $\phi(b)$ will actually contribute to the sum. According to the Nyquist-Shannon sampling theorem a sufficient condition for a discrete sequence of samples to capture all the information from the continuous function $\phi(b)$ is that $j_{\max }=\mathcal{N}$. Thus, regularization would demand writing

$$
\sum_{j=1}^{\infty} \rightarrow \lim _{A \rightarrow \infty} \sum_{j=1}^{A} \leq \lim _{\mathcal{N} \rightarrow \infty} \sum_{j=1}^{\mathcal{N}}
$$

for $A \in \mathbb{N}$ and $\mathcal{N} \geq A$. For finite values of $\mathcal{N}, j$, and $h$, the last term in inequality (B.9) will always be positive. Since regularization demands that $\mathcal{N} \geq A$, the inequality in (B.11) holds and so the term between parentheses in (B.10) will always converge to zero when $\mathcal{N} \rightarrow \infty$.

\section{One-dimensional convergence analysis for equidistant particles}

The error analysis developed in Section 3 can be tested for equidistant particles with spacing $\Delta$ over an infinite line so that $m / \rho=\Delta$. If, in addition, we consider the interpolation of the linear function $f(x)=\alpha+\beta x$ with the use of the Gaussian kernel

$$
W_{G}(x, h)=\frac{1}{\sqrt{\pi} h} \exp \left(-\frac{x^{2}}{h^{2}}\right),
$$

we can estimate the error in the SPH summation interpolant by calculating all terms in the series expansion on the right side of Eq. (13). For equidistant particles, Eq. (17) reduces to $x_{b}=b \Delta$. The SPH interpolation formula (9) gives, at $x_{a}=a \Delta$, the following approximation for $f(x)$

$$
f_{a}=\Delta \sum_{b=-\infty}^{\infty}(\alpha+\beta b \Delta) W(|a \Delta-b \Delta|, h) .
$$

If we shift the origin to the point $x_{a}=a \Delta$, make the change of variable $b=$ $q / \Delta$, and use the Poisson summation formula (13), the SPH approximation of the linear function becomes

$$
f_{a}=(\alpha+\beta a \Delta)\left[1+2 \sum_{j=1}^{\infty} \int_{-\infty}^{\infty} \cos \left(2 \pi j \frac{q}{\Delta}\right) W_{G}(q, h) d q\right] .
$$


Using the Gaussian kernel (C.1), Eq. (C.3) reduces to

$$
f_{a}=(\alpha+\beta a \Delta)\left[1+2 \sum_{j=1}^{\infty} \exp \left(-\frac{\pi^{2} j^{2} h^{2}}{\Delta^{2}}\right)\right]
$$

It is easy to show that the series expansion on the right side of Eq. (C.4) is absolutely convergent for all values of $h$ and $\Delta$. However, the SPH interpolation does not produce the linear function exactly even on a uniformly distributed set of particles unless $h / \Delta \rightarrow \infty$. However, if $h>\Delta$, the error becomes exponentially small.

Now, the integral on the right side of Eq. (C.3) can be integrated by parts to give

$$
\int_{-\infty}^{\infty} \cos \left(2 \pi j \frac{q}{\Delta}\right) W_{G}(q, h) d q=-\frac{\Delta}{\pi j} \int_{0}^{\infty} \sin \left(2 \pi j \frac{q}{\Delta}\right) \frac{\partial W_{G}}{\partial q} d q,
$$

which can be bounded as follows

$$
\begin{aligned}
\left|\int_{-\infty}^{\infty} \cos \left(2 \pi j \frac{q}{\Delta}\right) W_{G}(q, h) d q\right| & \leq \frac{\Delta}{\pi j}\left|\int_{0}^{\infty} \sin \left(2 \pi j \frac{q}{\Delta}\right) \frac{\partial W_{G}}{\partial q} d q\right| \\
& \leq \frac{\Delta}{\pi j}\left|\sin \left(2 \pi j \frac{q}{\Delta}\right)\right|\left|\int_{0}^{\infty} \frac{\partial W_{G}}{\partial q} d q\right| \\
& \leq \frac{\Delta}{\pi j} W_{G}(0, h)=\frac{\Delta}{\pi^{3 / 2} h j}
\end{aligned}
$$

after use of Eq. (C.1). Therefore, the error bound of the SPH interpolation (C.4) is

$$
\sum_{j=1}^{\infty} \exp \left(-\frac{\pi^{2} j^{2} h^{2}}{\Delta^{2}}\right) \leq \frac{\Delta}{\pi^{3 / 2} h} \sum_{j=1}^{\infty} \frac{1}{j} .
$$

Since the Gaussian kernel has infinite support, it is customary to define $\mathcal{N}$ as the average number of particles within distance $\sqrt{2} h$, which is directly related to the numerical resolution scale necessary for resolving sound waves in the continuum limit $h \gg d_{n}$ of large neighbor numbers, where $d_{n}$ is the nearest neighbor distance [45]. Then, it follows from Eq. (21) that $\Delta=2 \sqrt{2} h / \mathcal{N}$. Using this relation into the right side of inequality (C.7), regularizing the harmonic series in the sense of (B.11), and using Eq. (30) we obtain the 
asymptotic expansion for the error bound

$$
\sum_{j=1}^{\infty} \exp \left(-\frac{\pi^{2} j^{2} h^{2}}{\Delta^{2}}\right) \leq \frac{2 \sqrt{2}}{\pi^{3 / 2}} \lim _{\mathcal{N} \rightarrow \infty} \frac{1}{\mathcal{N}} \sum_{j=1}^{\mathcal{N}} \frac{1}{j} \leq \frac{2 \sqrt{2}}{\pi^{3 / 2}} \frac{(1+\gamma)}{\mathcal{N}}+O\left(\frac{1}{\mathcal{N}^{2}}\right)
$$

which converges to zero as $\mathcal{N} \rightarrow \infty$. This is consistent with the leftmost term converging exponentially to zero as $h / \Delta \rightarrow \infty$.

\section{Error bound for the kernel approximation in multidimensions}

An error bound for the kernel approximation in $n$-dimensions can be derived following essentially the same steps described in Appendix A for the one-dimensional case. The error made by the kernel approximation is given by the first summation on the right side of Eq. (36), which for convenience is written in the equivalent form

$$
\begin{aligned}
\mathcal{E}_{K S}^{(n)} & =\left\langle f\left(\mathbf{x}_{a}\right)\right\rangle-f\left(\mathbf{x}_{a}\right) \\
& +\frac{1}{2} \int_{\Omega_{n}}\left[\left(\mathbf{x}_{b}-\mathbf{x}_{a}\right) \cdot \nabla\right]^{2} f\left(\mathbf{x}_{a}\right) W\left(\left\|\mathbf{x}_{a}-\mathbf{x}_{b}\right\|, h\right) d^{n} \mathbf{x}_{b},
\end{aligned}
$$

where only terms up to $l=2$ are retained.

To find a bound on the error define

$$
\left[\left(\mathbf{x}_{b}-\mathbf{x}_{a}\right) \cdot \nabla\right]^{2} f(\xi)=[\mathbf{u} \cdot \nabla]^{2} f(\xi) \leq \sum_{i=1}^{n}\left[u_{i} \frac{\partial}{\partial \xi}\right]_{i}^{2} f\left(\xi_{i}\right) \leq n^{2} k^{2} h^{2}\left|D^{2} f(\xi)\right|
$$

where $\xi \in \Omega_{n}$ and $\mathbf{u}=\mathbf{x}_{b}-\mathbf{x}_{a}$. Moreover, defining

$$
e_{r}^{(2, n)}=\frac{k^{2} n^{2}}{2} \sup _{\xi \in \Omega_{n}}\left|D^{2} f(\xi)\right| \geq \frac{k^{2} n^{2}}{2}\left|D^{2} f(\xi)\right|,
$$

where the operator $D^{2}$ means any second-order derivative (pure or mixed), the error bound follows as

$$
\begin{aligned}
\left|\mathcal{E}_{K S}^{(n)}\right| & =\frac{1}{2}\left|\int_{\Omega_{n}}\left[\mathbf{x}_{b a} \cdot \nabla\right]^{2} f\left(\mathbf{x}_{a}\right) W\left(\left\|\mathbf{x}_{a b}\right\|, h\right) d^{n} \mathbf{x}_{b}\right| \\
& \leq \frac{1}{2} \int_{\Omega_{n}}\left|\left[\mathbf{x}_{b a} \cdot \nabla\right]^{2} f\left(\mathbf{x}_{\mathbf{a}}\right)\right| W\left(\left\|\mathbf{x}_{a b}\right\|, h\right) d^{n} \mathbf{x}_{b} \\
& \leq \frac{1}{2} n^{2} k^{2} h^{2}\left|D^{2} f(\xi)\right| \int_{\Omega_{n}} W\left(\left\|\mathbf{x}_{a b}\right\|, h\right) d^{n} \mathbf{x}_{b} \\
& \leq e_{r}^{(2, n)} h^{2}
\end{aligned}
$$


where $\mathbf{x}_{b a}=\mathbf{x}_{b}-\mathbf{x}_{a}$ and $\mathbf{x}_{a b}=-\mathbf{x}_{b a}$.

\section{E. Error bound for the particle approximation in multidimensions}

As mentioned in the main text, the error when passing from the kernel ap-

proximation to the particle approximation is quantified by the difference $\mathcal{E}_{K S}^{(n)}$ between the particle and the kernel estimates of a function $f(\mathbf{x})$ evaluated at the interpolation point $\mathbf{x} \in \mathbb{R}^{n}$. For a given particle $a$ at $\mathbf{x}_{a}$, this difference is given by the double summation in Eq. (36), which for convenience is written in the equivalent form

$$
\mathcal{E}_{K S}^{(n)}=\sum_{\substack{\mathbf{j} \in \Lambda^{*} \\ \mathbf{j} \neq 0}}^{\infty} \sum_{l=0}^{\infty} \frac{1}{l !} \int_{\Omega_{n}}\left[\left(\mathbf{x}_{b}-\mathbf{x}_{a}\right) \cdot \nabla\right]^{l} f\left(\mathbf{x}_{a}\right) W_{a b} \exp (-i 2 \pi \mathbf{j} \cdot \mathbf{b}) d^{n} \mathbf{x}_{b},
$$

where $W_{a b}=W\left(\left\|\mathbf{x}_{a}-\mathbf{x}_{b}\right\|, h\right)$. To obtain a bound on this error define

$$
\left[\left(\mathbf{x}_{b}-\mathbf{x}_{a}\right) \cdot \nabla\right]^{l} f(\xi)=[\mathbf{u} \cdot \nabla]^{l} f(\xi) \leq \sum_{i}^{n}\left[\left|u_{i} \frac{\partial}{\partial \xi_{i}}\right|\right]^{l} f\left(\xi_{i}\right) \leq n^{l} k^{l} h^{l}\left|D^{l} f(\xi)\right|,
$$

where $\xi \in \Omega_{n}, \mathbf{u}=\mathbf{x}_{b}-\mathbf{x}_{a}$, and $D^{l}$ denotes any $l$ th-order pure or mixed derivative. Now defining

$$
\tilde{e}_{r}^{(l, n)}=\frac{k^{l} n^{l}}{l !} \sup _{\xi \in \Omega_{n}}\left|D^{l} f(\xi)\right| \geq \frac{k^{l} n^{l}}{l !}\left|D^{l} f(\xi)\right|,
$$

and the bound of the kernel function as

$$
W\left(\left\|\mathbf{x}_{a}-\mathbf{x}_{b}\right\|, h\right) \leq \frac{a_{0}}{h^{n}},
$$

where $a_{0}>0$, the bound on $\mathcal{E}_{K S}^{(n)}$ can be calculated as follows

$$
\begin{aligned}
\left|\mathcal{E}_{K S}^{(n)}\right| & =\left|\sum_{\substack{\mathbf{j} \in \Lambda^{*} \\
\mathbf{j} \neq \mathbf{0}}}^{\infty} \sum_{l=0}^{\infty} \frac{1}{l !} \int_{\Omega_{n}}\left[\mathbf{x}_{b a} \cdot \nabla\right]^{l} f\left(\mathbf{x}_{a}\right) W_{a b} \exp (-i 2 \pi \mathbf{j} \cdot \mathbf{b}) d^{n} \mathbf{x}_{b}\right| \\
& \leq \sum_{\substack{\mathbf{j} \in \Lambda^{*} \\
\mathbf{j} \neq \mathbf{0}}}^{\infty} \sum_{l=0}^{\infty} \frac{1}{l !}\left|\int_{\Omega_{n}}\left[\mathbf{x}_{b a} \cdot \nabla\right]^{l} f\left(\mathbf{x}_{a}\right) W_{a b} \exp (-i 2 \pi \mathbf{j} \cdot \mathbf{b}) d^{n} \mathbf{x}_{b}\right|,(\mathbb{E},)
\end{aligned}
$$


where $W_{a b}=W\left(\left\|\mathbf{x}_{a b}\right\|, h\right), \mathbf{x}_{a b}=\mathbf{x}_{a}-\mathbf{x}_{b}$, and $\mathbf{x}_{a b}=-\mathbf{x}_{b a}$. Let $\mathbf{x}_{b, \max } \in$ $\Omega_{n}$ define a point where $\left[\mathbf{x}_{b, \max , a} \cdot \nabla\right]^{l} f\left(\mathbf{x}_{a}\right) W\left(\left\|\mathbf{x}_{a b, \max }\right\|, h\right)=\max \left\{\left[\mathbf{x}_{b a}\right.\right.$. $\left.\nabla]^{l} f\left(\mathbf{x}_{a}\right) W\left(\left\|\mathbf{x}_{a b}\right\|, h\right)\right\}$ and $\mathbf{x}_{a b, \max }=\mathbf{x}_{a}-\mathbf{x}_{b, \max }$. Applying the CauchySchwarz inequality to (E.5) yields

$$
\begin{aligned}
\left|\mathcal{E}_{K S}^{(n)}\right| & \leq \sum_{\substack{\mathbf{j} \in \Lambda^{\star} \\
\mathbf{j} \neq \mathbf{0}}}^{\infty} \sum_{l=0}^{\infty} \frac{1}{l !}\left|\left[\mathbf{x}_{b, \max , a} \cdot \nabla\right]^{l} f\left(\mathbf{x}_{a}\right)\right| W_{a b, \max }\left|\int_{\Omega_{n}} \exp (-i 2 \pi \mathbf{j} \cdot \mathbf{b}) d^{n} \mathbf{x}_{b}\right| \\
& \leq a_{0} \sum_{l=0}^{\infty} h^{l-n} \tilde{e}_{r}^{(l, n)} \sum_{\substack{\mathbf{j} \in \Lambda^{\star} \\
\mathbf{j} \neq \mathbf{0}}}^{\infty}\left|\int_{\Omega_{n}} \exp (-i 2 \pi \mathbf{j} \cdot \mathbf{b}) d^{n} \mathbf{x}_{b}\right|
\end{aligned}
$$

where $W_{a b, \max }=W\left(\left\|\mathbf{x}_{a b, \text { max }}\right\|, h\right)$ and relation (E.3) has been used together with the definitions $\left|\mathbf{x}_{b, \max , a}\right| \leq k^{l} h^{l}$ and $W\left(\left\|\mathbf{x}_{a b}\right\|, h\right) \leq a_{0} / h^{n}$.

A bound to the Fourier integral when $\mathcal{N} \gg 1$ can be found by expanding the vector function $\mathbf{b}\left(\mathbf{x}_{b}\right)$ about $\mathbf{x}_{a}$ to yield

$$
\mathbf{b}\left(\mathbf{x}_{b}\right)=\mathbf{b}\left(\mathbf{x}_{a}\right)+\mathbf{J}_{\mathbf{x}_{b}}\left(\mathbf{x}_{a}\right) \cdot\left(\mathbf{x}_{b}-\mathbf{x}_{a}\right)+O\left(\left\|\mathbf{x}_{b}-\mathbf{x}_{a}\right\|^{2}\right)
$$

for $\mathbf{x}_{b}$ close to $\mathbf{x}_{a}$, where $\left\|\mathbf{x}_{b}-\mathbf{x}_{a}\right\|$ is the distance between $\mathbf{x}_{b}$ and $\mathbf{x}_{a}$ in the $n$-dimensional Euclidean space. Since this expression defines a linear mapping $\mathbb{R}^{n} \rightarrow \mathbb{R}^{m}$ with $m=n$, the determinant of the Jacobian matrix $\mathbf{J}_{\mathbf{x}_{b}}\left(\mathbf{x}_{a}\right)$ is given by relation (35), which from use of Eqs. (39) and (41) can be written as

$$
\left|\mathbf{J}_{\mathbf{x}_{b}}\left(\mathbf{x}_{a}\right)\right|=\frac{\rho\left(\mathbf{x}_{a}\right)}{m\left(\mathbf{x}_{a}, h\right)}=\frac{n \mathcal{N}}{B_{n} k^{n} h^{n}}=\frac{\mathcal{N}}{\mathcal{V}_{n}}=\frac{1}{\Delta_{m}^{n}},
$$

for $k h \ll 1$, where $\mathcal{V}_{n}=B_{n} k^{n} h^{n} / n$ is the $n$-dimensional volume of the kernel support and $\Delta_{m}$ is the mean distance of particle pairs within the kernel support. Note that for low-discrepancy sequences of sample points $\Delta_{m} \approx \Delta_{s}$, where $\Delta_{s}$ (for $\left.s=1,2, \ldots, n\right)$ is the projected mean distance on the sth-axis of an $n$-dimensional Cartesian coordinate system. Use of the above linear mapping then yields for $\mathbf{j} \cdot \mathbf{b}$ the expression

$$
\mathbf{j} \cdot \mathbf{b}\left(\mathbf{x}_{b}\right)=\mathbf{j} \cdot \mathbf{b}\left(\mathbf{x}_{a}\right)+\mathbf{j}^{T} \cdot \mathbf{J}_{\mathbf{x}_{b}}\left(\mathbf{x}_{a}\right) \cdot\left(\mathbf{x}_{b}-\mathbf{x}_{a}\right),
$$

where $\mathbf{j}^{T}$ is a row vector and $\mathbf{x}_{b}-\mathbf{x}_{a}$ is a column vector. Defining the vector $\mathbf{v}$ as

$$
\mathbf{v}=\mathbf{J}_{\mathbf{x}_{b}}\left(\mathbf{x}_{a}\right) \cdot\left(\mathbf{x}_{b}-\mathbf{x}_{a}\right)
$$


and noting that $d^{n} \mathbf{v}=\left[n \mathcal{N} /\left(B_{n} k^{n} h^{n}\right)\right] d^{n} \mathbf{x}_{b}$ by Eq. (E.8), the Fourier integral in inequality (E.6) becomes

$$
\begin{aligned}
\int_{\Omega_{n}} \exp (-i 2 \pi \mathbf{j} \cdot \mathbf{b}) d^{n} \mathbf{x}_{b} & =\exp \left[-i 2 \pi \mathbf{j} \cdot \mathbf{b}\left(\mathbf{x}_{a}\right)\right] \frac{B_{n} k^{n} h^{n}}{n \mathcal{N}} \\
& \times \int_{\tilde{\Omega}_{n}} \exp (-i 2 \pi \mathbf{j} \cdot \mathbf{v}) d^{n} \mathbf{v}
\end{aligned}
$$

where $\tilde{\Omega}=\tilde{\Omega}(\mathbf{v}, h)$ is the image domain of $\Omega=\Omega\left(\mathbf{x}_{b}, h\right)$ due to the mapping $\mathbf{x}_{b} \rightarrow \mathbf{v}$. If the Fourier exponential oscillates rapidly within the domain $\Omega_{n}$, then the Fourier integral in (E.11) vanishes. For $\mathcal{N} \gg 1$, this condition allows to test its convergence to zero. Now, expressing $\mathbf{j} \cdot \mathbf{b}$ in component form and noting that $d^{n} \mathbf{v}=d v_{1} d v_{2} \cdots d v_{n}$, the integral on the right side of (E.11) can be rewritten as

$$
\begin{aligned}
\int_{\tilde{\Omega}_{n}} \exp (-i 2 \pi \mathbf{j} \cdot \mathbf{v}) d^{n} \mathbf{v} & =\int_{\tilde{\Omega}_{n}} \exp \left(-i 2 \pi \sum_{s=1}^{n} j_{s} v_{s}\right) d^{n} \mathbf{v} \\
& =\prod_{s=1}^{n} \int_{-c}^{c} \exp \left(-i 2 \pi j_{s} v_{s}\right) d v_{s}
\end{aligned}
$$

where $j_{s}$ and $v_{s}$ are, respectively, the projections of vectors $\mathbf{j}$ and $\mathbf{v}$ on the $s$ th-axis of an $n$-dimensional Cartesian coordinate system. The limits of integration in the second equality of Eq. (E.12) can be estimated as follows. First, calculate a bound on the magnitude of vector $\mathbf{v}$ from Eq. (E.10) as

$$
v \leq k h\left\|\mathbf{J}_{\mathbf{x}_{b}}\left(\mathbf{x}_{a}\right)\right\|_{\infty}=k h \max _{i} \sum_{j=1}^{n}\left|J_{i j}\right|=\frac{k h}{\Delta_{m}} \approx \frac{k h}{\Delta_{s}}
$$

where $\left\|\mathbf{J}_{\mathbf{x}_{b}}\left(\mathbf{x}_{a}\right)\right\|_{\infty}$ is the max norm of the Jacobian matrix defined as the maximum absolute row sum of its elements $J_{i j}$. Note that the last equality in Eq. (E.13) holds because according to Eq. (E.8) the Jacobian is a diagonal matrix with elements $J_{i i}=1 / \Delta_{m}$. Since $\Delta_{m} \approx \Delta_{s}$ for a low-discrepancy sequence of sample points, it follows that the projected components of vector $\mathbf{v}$ on the $s$ th-axis of the $n$-dimensional Cartesian coordinate system satisfy the bound inequality $\left|v_{s}\right| \leq c=k h_{s} / \Delta_{s}$, where $h_{s}$ is the projection of $h$ on the sth-axis of the Cartesian system. Following similar steps to those described in Appendix B for the one-dimensional case, it is easy to show 
that

$$
\left|\int_{-c}^{c} \exp \left(-i 2 \pi j_{s} v_{s}\right) d v_{s}\right|=\left|\int_{-c}^{c} \cos \left(2 \pi j_{s} v_{s}\right) d v_{s}\right| \leq \frac{1}{\pi j_{s}}
$$

Since the maximum value of the cosine integral is $1 / \pi j_{s}$, the bound on the Fourier integral takes the form

$$
\sum_{\substack{\mathbf{j} \in \Lambda^{\star} \\ \mathbf{j} \neq \mathbf{0}}}^{\infty}\left|\int_{\Omega_{n}} \exp (-i 2 \pi \mathbf{j} \cdot \mathbf{b}) d^{n} \mathbf{x}_{b}\right| \leq\left(\frac{2}{\pi}\right)^{n} \frac{B_{n} k^{n} h^{n}}{n}\left(\lim _{\mathcal{N} \rightarrow \infty} \frac{1}{\mathcal{N}} \prod_{s=1}^{n} \sum_{j_{s}=1}^{\mathcal{N}_{s}} \frac{1}{j_{s}}\right),
$$

where $\mathcal{N}_{s}=\left[2 k h_{s} / \Delta_{s}\right]$. With this result, the bound on $\mathcal{E}_{K S}^{(n)}$ can be finally written as

$$
\left|\mathcal{E}_{K S}^{(n)}\right| \leq\left(\frac{2}{\pi}\right)^{n} \frac{a_{0} B_{n} k^{n}}{n} \sum_{l=0}^{\infty} h^{l} \tilde{e}_{r}^{(l, n)}\left(\lim _{\mathcal{N} \rightarrow \infty} \frac{1}{\mathcal{N}} \prod_{s=1}^{n} \sum_{j_{s}=1}^{\mathcal{N}_{s}} \frac{1}{j_{s}}\right),
$$

which demonstrates inequality (43). Noting that $\Delta_{s} \approx \Delta_{\mathrm{m}}$ for a low-discrepancy set of sample points, it follows from relation (41) that $\mathcal{N}_{s} \propto \mathcal{N}^{1 / n}$ and hence the limit between parentheses in inequality (E.16) converges in the sense of (B.11).

\section{Acknowledgement}

We are grateful to the anonymous reviewers for their valuable comments and suggestions. This work was partially supported by the Conacyt ENERXICO project under the grant number B-S-6992, the Division of Basic Sciences and Engineering (CBI) of the Autonomous Metropolitan University (UAM-A) and the Venezuelan Institute of Scientific Research (IVIC) through internal funds. The calculations of this paper were performed using the computing facilities of Cinvestav-Abacus.

\section{References}

\section{References}

[1] L. B. Lucy, A numerical approach to the testing of the fission hypothesis, Astron. J. 82 (1977) 1013-1024. 
[2] R. A. Gingold, J. J. Monaghan, Smoothed particle hydrodynamics theory and application to non-spherical stars, Mon. Not. R. Astron. Soc. 181 (1977) 375-389.

[3] J. J. Monaghan, Smoothed particle hydrodynamics, Rep. Progr. Phys. 68 (8) (2005) 1703-1759.

[4] S. Rosswog, Astrophysical smooth particle hydrodynamics, New Astron. Rev. 33 (2009) 78-104.

[5] M. B. Liu, G. R. Liu, Smoothed particle hydrodynamics (sph): An overview and recent developments, Arch. Comput. Methods Eng. 17 (2010) 25-76.

[6] J. J. Monaghan, Smoothed particle hydrodynamics and its diverse applications, Annu. Rev. Fluid Mech. 44 (2012) 323-346.

[7] J. Bonet, T.-S. L. Lok, Variational and momentum preservation aspects of smooth particle hydrodynamics formulations, Comput. Meth. Appl. Mech. Eng. 180 (1999) 97-115.

[8] J. K. Chen, J. E. Beraun, C. J. Jih, Completeness of corrective smoothed particle method for linear elastodynamics, Comput. Mech. 24 (1999) 273-285.

[9] M. B. Liu, G. R. Liu, K. Y. Lam, Constructing smoothing functions in smoothed particle hydrodynamics with applications, J. Comput. Appl. Math. 155 (2003) 263-284.

[10] G. M. Zhang, R. C. Batra, Modified smoothed particle hydrodynamics method and its application to transient problems, Comput. Mech. 34 (2004) 137-146.

[11] M. B. Liu, G. R. Liu, Restoring particle consistency in smoothed particle hydrodynamics, Appl. Numer. Math. 56 (2006) 19-36.

[12] S. Litvinov, X. Y. Hu, N. A. Adams, Towards consistence and convergence of conservative sph approximations, J. Comput. Phys. 301 (2015) 394-401.

[13] S. Sibilla, An algorithm to improve consistency in smoothed particle hydrodynamics, Comput. Fluids 118 (2015) 148-158. 
[14] B. B. Moussa, J. P. Vila, Convergence of sph method for scalar nonlinear conservation laws, SIAM J. Numer. Anal. 37 (3) (2000) 863-887.

[15] N. J. Quinlan, M. Basa, M. Lastiwka, Truncation error in mesh-free particle methods, Int. J. Numer. Meth. Eng. 66 (2006) 2064-2085.

[16] G. L. Vaughan, T. R. Healy, K. R. Bryan, A. D. Sneyd, R. M. Gorman, Completness, conservation and error in sph for fluids, Int. J. Numer. Meth. Eng. 56 (2008) 37-62.

[17] J. I. Read, T. Hayfield, O. Agertz, Resolving mixing in smoothed particle hydrodynamics, Mon. Not. R. Astron. Soc. 405 (2010) 1513-1530.

[18] R. Fatehi, M. T. Manzari, Error estimation in smoothed particle hydrodynamics and a new scheme for second derivatives, Comput. Math. Appl. 61 (2011) 482-498.

[19] Q. Zhu, L. Hernquist, Y. Li, Numerical convergence in smoothed particle hydrodynamics, Astrophys. J. 800 (1) (2015) id. 6, 13pp.

[20] R. D. Lisio, E. Grenier, M. Pulvirenti, On the regularization of the pressure field in compressible euler equations, Ann. Sc. Norm. Super. Pisa Cl. Sci. (5) 24 (2) (1997) 227-238.

[21] R. D. Lisio, E. Grenier, M. Pulvirenti, The convergence of the sph method, Comput. Math. Appl. 35 (1-2) (1998) 95-102.

[22] I. A. Zisis, J. van der Linden, J. H. M. Evers, M. H. Duong, Recent results in the systematic derivation and convergence of sph, arXiv preprint arXiv: 1612.06687v1 [math.NA].

[23] S. Adami, X. Y. Hu, N. A. Adams, A transport-velocity formulation for smoothed particle hydrodynamics, J. Comput. Phys. 241 (2013) 292307.

[24] A. Krimi, M. Rezoug, S. Khelladi, X. Nogueira, M. Deligant, L. Ramírez, Smoothed particle hydrodynamics: A consistent model for interfacial multiphase fluid flow simulations, J. Comput. Phys. 358 (2018) 53-87.

[25] T. Belytschko, Y. Y. Lu, L. Gu, Element-free Galerkin methods, Int. J. Numer. Meth. Eng. 37 (1994) 229-256. 
[26] W. K. Liu, S. Jun, Y. Zhang, Reproducing kernel particle methods, Int. J. Numer. Meth. Eng. 40 (1995) 1081-1106.

[27] W. K. Liu, S. Li, T. Belytschko, Moving least square reproducing kernel methods (I) Methodology and convergence, Comput. Meth. Appl. Mech. Eng. 143 (1-2) (1997) 113-154.

[28] H. M. Shodja, A. Hashemian, A remedy to gradient type constraint dilemma encountered in RKPM, Adv. Eng. Software 38 (2007) 229-243.

[29] A. Hashemian, H. M. Shodja, Gradient reproducing kernel particle method, J. Mech. Mat. Strut. 3 (1) (2008) 127-152.

[30] S. N. Atluri, J. Y. Cho, H. G. Kim, Analysis of thin beams using local Petrov-Galerkin method with generalized moving least squares interpolations, Comput. Mech. 24 (1999) 334-347.

[31] I. V. Singh, P. K. Jain, Parallel meshless EFG solution for fluid flow problems, Numer. Heat Transfer, Part B: Fundamentals 48 (1) (2005) 45-66.

[32] R. Staroszczyk, Application of an element-free Garlekin method to water wave propagation problems, Arch. Hydro-Eng. Environ. Mech. 60 (1-4) (2013) 87-105.

[33] K. Kiani, Characterization of free vibartion of elastically supported double-walled carbon nanotubes subjected to a longitudinally varying magnetic field, Acta Mech. 224 (12) (2013) 3139-3151.

[34] K. Kiani, A nonlocal meshless solution for flexural vibrations of doublewalled carbon nanotubes, Appl. Math. Comput. 234 (2014) 557-578.

[35] K. Kiani, Nonmechanical sensors based on elastically supported doublewalled carbon nanotubes, Appl. Math. Comput. 270 (2015) 216-241.

[36] H. M. Shodja, K. Kiani, A. Hashemian, A model for the evolution of concrete deterioration due to reinforcement corrosion, Math. Comput. Modelling 52 (2010) 1403-1422.

[37] K. Kiani, A. Nikkhoo, B. Mehri, Parametric analyses of multispan viscoelastic shear deformable beams under excitation of a moving mass, J. Vib. Acoust. 131 (5) (2009) 051009. 
[38] K. Kiani, A. Nikkhoo, B. Mehri, Assessing dynamic response of multispan viscoelastic thin beams under a moving mass via generalized moving least square method, Acta Mech. Sin. 26 (2010) 721-733.

[39] L. Schwartz, Théorie des Distributions, Hermann, Paris, 1966.

[40] L. D. G. Sigalotti, J. Klapp, O. Rendón, C. A. Vargas, F. Peña-Polo, On the kernel and particle consistency in smoothed particle hydrodynamics, Appl. Numer. Math. 108 (2016) 242-255.

[41] D. A. Fulk, A numerical analysis of smoothed particle hydrodynamics, Phd thesis, School of Engineering of the Air Force Institute of Technology, Air University, Montgomery, Alabama, USA (1994).

[42] J. J. Monaghan, Particle methods for hydrodynamics, Comput. Phys. Rep. 3 (1985) 71-124.

[43] F. A. Rasio, Particle methods in astrophysical fluid dynamics, Progr. Theoret. Phys. Suppl. 138 (2000) 609-621.

[44] H. Wendland, Piecewise polynomial, positive definite and compactly supported radial functions of minimal degree, Adv. Comput. Math. 4 (1995) 389-396.

[45] W. Dehnen, H. Aly, Improving convergence in smoothed particle hydrodynamics simulations without pairing instability, Mon. Not. R. Astron. Soc. 425 (2) (2012) 1068-1082.

[46] Y. F. Meyer, Measures with locally finite support and spectrum, Proc. Nat. Acad. Sci. 113 (12) (2016) 3152-3158.

[47] R. M. Young, Euler's constant, Math. Gaz. 75 (472) (1991) 187-190.

[48] H. Woźniakowski, Average case complexity of multivariate integration, Bull. Am. Math. Soc. 24 (1) (1991) 185-193.

[49] J. J. Monaghan, Smoothed particle hydrodynamics, Annu. Rev. Astron. Astrophys. 30 (1992) 543-574.

[50] J. H. Wilkinson, Rounding Errors in Algebraic Processes, Dover Publications, New York, 1994. 
Figure 1: Dependence of the 2D (solid circles) and 3D (solid squares) error bounds given by relations (56) and (57), respectively, and the mean absolute errors (MAEs) between the SPH estimates of the test functions (53) (open circles) and (54) (open squares) and their exact values on spatial resolution $N^{1 / n}$, where $n=2$ in $2 \mathrm{D}$ and $n=3$ in $3 \mathrm{D}$.

Figure 2: Distributions of the volume estimate $M_{0}$ for the 2D quasi-random point set for the spatial resolutions listed in Table 1 . The distributions of $M_{0}$ slowly approach a narrow normal distribution peaking at $M_{0}=1$ with increasing $N$ and $\mathcal{N}$. The left inset depicts the maximum of the distributions as a function of $N^{1 / 2}$ and the right inset shows the details of the distributions for $N \leq 100^{2}$. This quantifies the deviation from an exact partition of unity.

Figure 3: Distributions of the volume estimate $M_{0}$ for the 3D quasi-random point set for the spatial resolutions listed in Table 1 . The distributions of $M_{0}$ slowly approach a narrow normal distribution peaking at $M_{0}=1$ with increasing $N$ and $\mathcal{N}$. The left inset depicts the maximum of the distributions as a function of $N^{1 / 3}$ and the right inset shows the details of the distributions for $N \leq 100^{3}$. This quantifies the deviation from an exact partition of unity. 


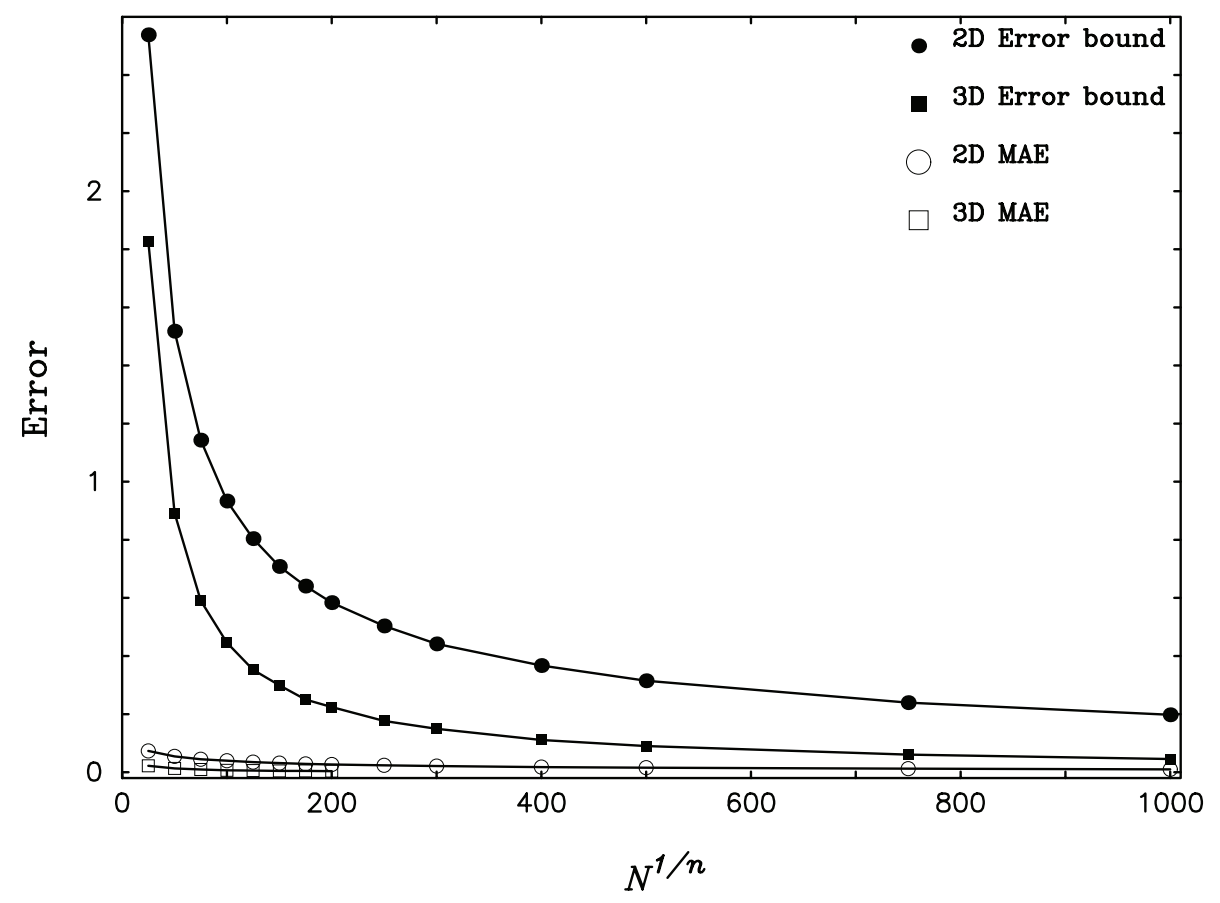

Figure 1 


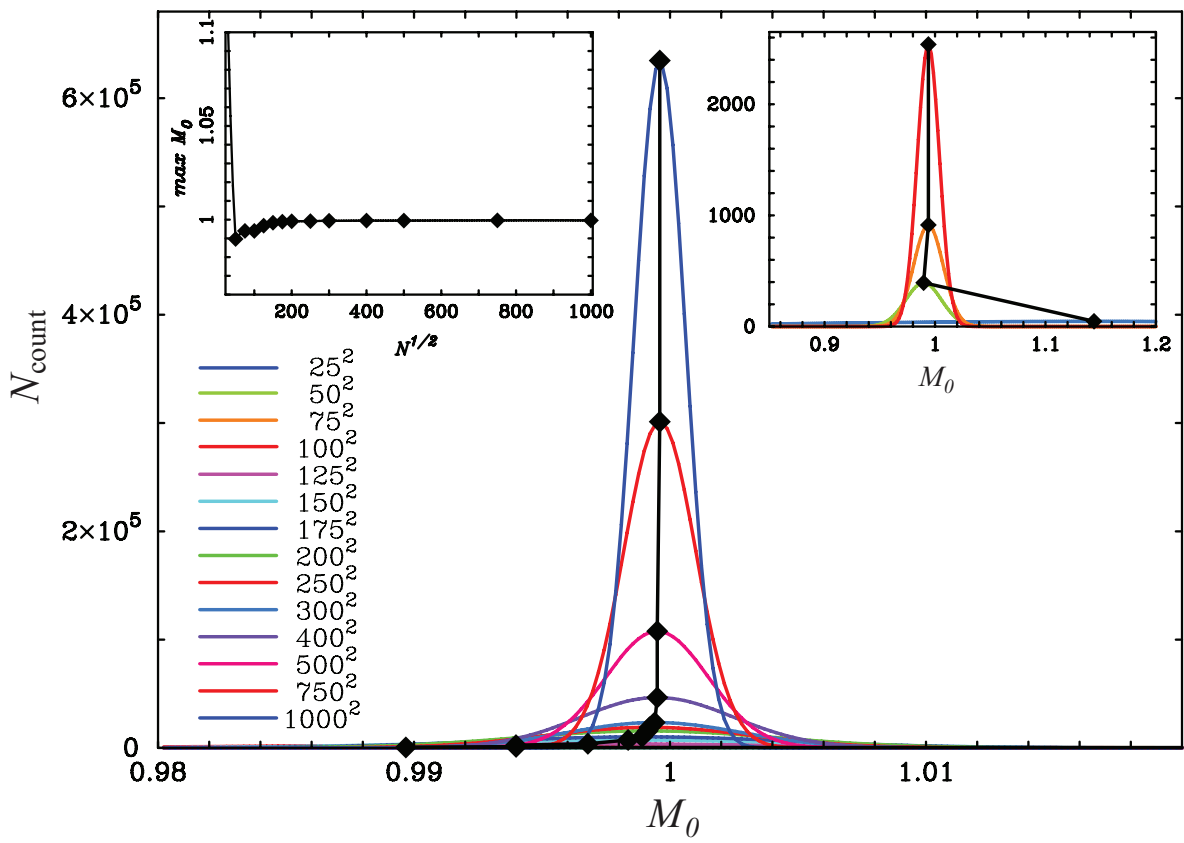

Figure 2 


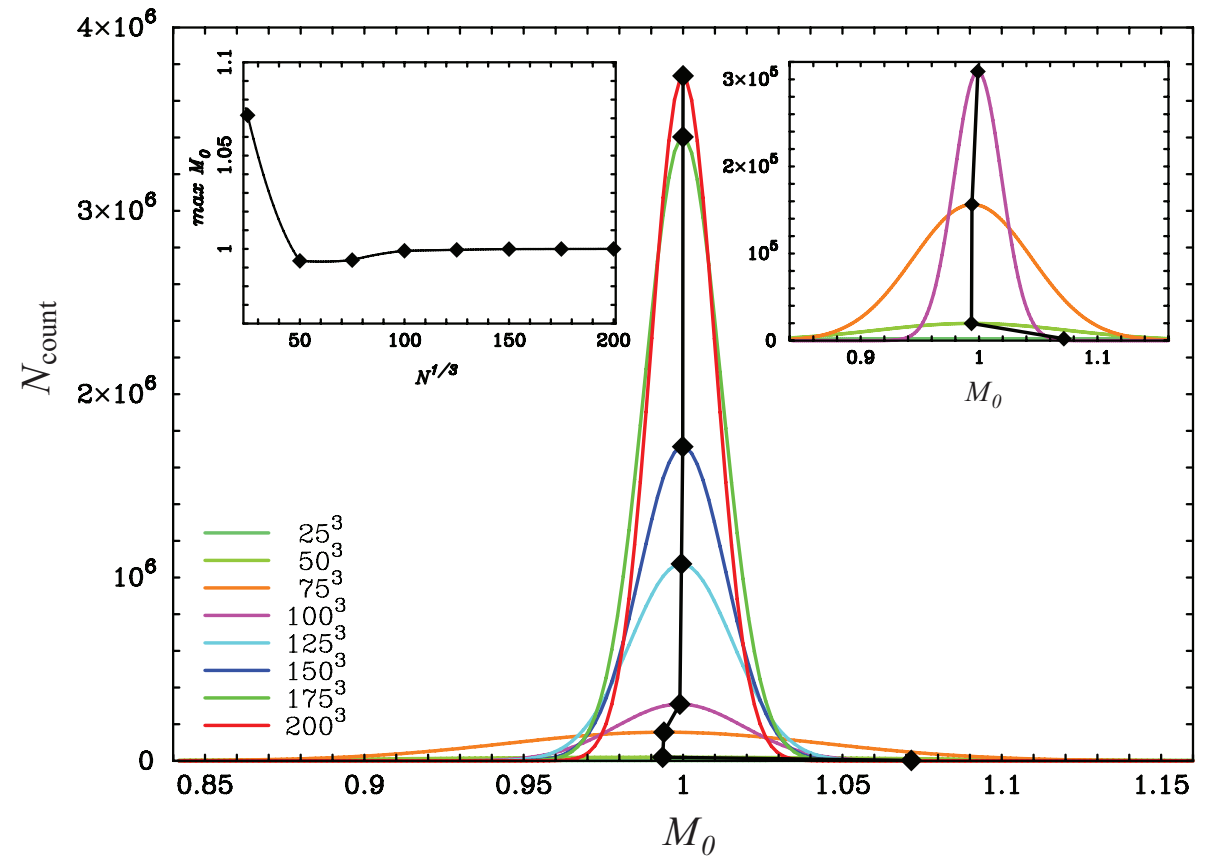

Figure 3 